PHYSICAL REVIEW D 95, 094503 (2017)

\title{
Higher order quark number fluctuations via imaginary chemical potentials in $N_{f}=2+1$ QCD
}

\author{
Massimo D'Elia ${ }^{*}$ and Giuseppe Gagliardi ${ }^{\dagger}$ \\ Dipartimento di Fisica dell'Università di Pisa and INFN_Sezione di Pisa, \\ Largo Pontecorvo 3, I-56127 Pisa, Italy
}

Francesco Sanfilippo

School of Physics and Astronomy, University of Southampton, SO17 1BJ Southampton, United Kingdom (Received 16 December 2016; published 11 May 2017)

\begin{abstract}
We discuss analytic continuation as a tool to extract the cumulants of the quark number fluctuations in the strongly interacting medium from lattice QCD simulations at imaginary chemical potentials. The method is applied to $N_{f}=2+1 \mathrm{QCD}$, discretized with stout improved staggered fermions, physical quark masses and the tree level Symanzik gauge action, exploring temperatures ranging from 135 up to $350 \mathrm{MeV}$ and adopting mostly lattices with $N_{t}=8$ sites in the temporal direction. The method is based on a global fit of various cumulants as a function of the imaginary chemical potentials. We show that it is particularly convenient to consider cumulants up to order two, and that below $T_{c}$ the method can be advantageous, with respect to a direct Montecarlo sampling at $\mu=0$, for the determination of generalized susceptibilities of order four or higher, and especially for mixed susceptibilities, for which the gain is well above one order of magnitude. We provide cumulants up to order eight, which are then used to discuss the radius of convergence of the Taylor expansion and the possible location of the second-order critical point at real $\mu$ : no evidence for such a point is found in the explored range of $T$ and for chemical potentials within present determinations of the pseudocritical line.
\end{abstract}

DOI: $10.1103 /$ PhysRevD.95.094503

\section{INTRODUCTION}

The study of the QCD phase diagram at finite temperature and density is presently a challenging problem. Its comprehension is particularly relevant, from a phenomenological point of view, to the physics of compact stars and to understanding the properties of the strongly interacting medium formed in heavy ion collisions. One outstanding open issue is related to the nature of the deconfinement transition, which is known to be an analytic crossover at zero baryon density [1]; it has been suggested that it could become a true transition at some critical value of the baryon chemical potential $\mu_{B}$, which would represent the second-order critical end point (CEP) of a line of firstorder transitions existing for larger values of $\mu_{B}$.

Even if indications for the existence of a CEP are given by many effective models, clear evidence from the first principles, in favor or against its existence, are still lacking. Indeed, at present, a direct lattice evaluation of the QCD equation of state at finite $\mu_{B}$ is hindered by the well-known sign problem: the Euclidean path integral measure becomes

\footnotetext{
*massimo.delia@unipi.it

Present address: Universität Bielefeld, Fakultät für Physik, D-33615 Bielefeld, Germany. giuseppe@physik.uni-bielefeld.de

${ }^{\ddagger}$ Present address: INFN, Sez. di Roma Tre, Via della Vasca Navale 84, I-00146 Rome, Italy.

f.sanfilippo@soton.ac.uk
}

complex in the presence of quark chemical potentials, making it impossible to apply ordinary Monte Carlo algorithms based on the interpretation of the measure as a probability density.

One way to partially overcome the problem is the Taylor expansion technique. Assuming analyticity around $\mu=0$, the free energy $F$ can be expanded in powers of the quark chemical potentials. Let us consider, for instance, the case of QCD with three flavors of quarks (u, d, s); the expansion is given by

$$
\begin{aligned}
& F\left(T, V, \mu_{u}, \mu_{d}, \mu_{s}\right) \\
& \quad=F(T, V, 0)+V T^{4} \sum_{i+j+k=\text { even }} \frac{\chi_{i j k}(T)}{i ! j ! k !} \hat{\mu}_{u}^{(i)} \hat{\mu}_{d}^{(j)} \hat{\mu}_{s}^{(k)},
\end{aligned}
$$

where $V$ is the spatial volume, $\hat{\mu}_{q} \equiv \mu_{q} / T$ and odd monomials are zero due to the symmetry under charge conjugation of the theory at zero chemical potentials. The coefficients

$$
\chi_{i j k}(T)=\left.\frac{1}{V T^{4}} \frac{\partial^{(i+j+k)} F(T, \mu)}{\partial \hat{\mu}_{u}^{(i)} \partial \hat{\mu}_{d}^{(j)} \partial \hat{\mu}_{s}^{(k)}}\right|_{\mu_{u}=\mu_{d}=\mu_{s}=0}
$$

are usually known as the "generalized quark number susceptibilities" and can be computed by means of standard Monte Carlo algorithms at zero chemical potentials. 
The interest in generalized susceptibilities $\chi_{i j k}(T, \mu)$ has increased over the last few years: cumulants of conserved charges, i.e. baryon number $B$, electric charge $Q$ and strangeness $S$ are directly accessible in heavy-ion collision experiments by evaluating their event-by-event fluctuations [2-5]. They can be related to the derivatives of the free energy with respect to $\mu_{B}, \mu_{Q}$ and $\mu_{S}$, which in turn can be obtained as a linear combination of the coefficients $\chi_{i j k}$. It has been shown [6-8] that comparing the experimental measure of these cumulants with lattice QCD computations, it is possible to extract the freeze-out parameters without relying on phenomenological models such as the hadron resonances gas model (HRG), thus directly from the first principles of the theory. Moreover, the cumulants represent a sensitive probe of the possible critical behavior associated with the CEP: the knowledge of a large enough number of terms in the Taylor expansion in Eq. (1) would allow to obtain an estimate of the radius of convergence of the series, hence of the location of the CEP [9-14].

However, a direct lattice computation of the generalized susceptibilities suffers from at least two problems, which combine to make the numerical effort increase exponentially with the order of the susceptibility:

(i) The calculation of each $\chi_{i j k}(T, 0)$ requires the evaluation of a number of different terms which rapidly increases with the order. In particular, an increasing number of inversions of the Euclidean Dirac operator $(\not D+m)$ is required, which represent the most time consuming part of the computation;

(ii) The direct sampling of nonlinear susceptibilities suffers from the so-called problem of lacking of self-averaging [15]: the signal-to-noise ratio of these quantities decreases as a power law of the spatial volume $V$, with an exponent that grows with the order of the susceptibility. This is essentially a consequence of the central limit theorem: the determination of nonlinear susceptibilities involves the measurement of deviations from a Gaussian distribution, but in the large volume limit, and away from criticality, the distribution of the quark number, $N_{q}$, can be well approximated by a Gaussian of variance $V \chi_{2}$, with deviations which are suppressed as powers of the volume.

For instance, from Eq. (1), it follows that $\left(V \chi_{2 n}\right)$ are extensive quantities, i.e. scaling linearly with the spatial volume; however, a direct computation shows that they are formed of a combination of terms such as, $\left\langle N_{q}^{2 n}\right\rangle$, $\left\langle N_{q}^{2(n-1)}\right\rangle\left\langle N_{q}^{2}\right\rangle, \ldots\left\langle N_{q}^{2}\right\rangle^{n}$, which in the large volume limit grow like $\left(\chi_{2} V\right)^{n}$, up to subleading powers of $V$. However, it is precisely after a fine cancellation of such terms that the subleading corrections produce the final signal, which scales like $V$, inducing a signal-to-noise-ratio scaling as $V^{(1-n)}$. This implies that to achieve a given statistical accuracy for the $2 n$ th-order susceptibility on a certain range of volumes, the number of sampled gauge configurations should be increased proportionally to $V^{2(n-1)}$.

As a consequence, the computation of the Taylor series in Eq. (1) becomes a hard numerical challenge, when one tries to increase the order of the expansion. It is possible to reduce this problem by inserting explicit external sources directly coupled to the quark number operators, then exploiting the fact that the dependence of lower-order cumulants on the external sources contains useful information about the higher-order cumulants: this is analogous to determining the magnetic susceptibility of a material from an analysis of the magnetization as a function of the external magnetic field, rather than from measuring fluctuations at zero external field. In our case, the external sources to be used are the chemical potentials. Given that real-valued chemical potentials lead to a sign problem, one can perform numerical simulations at purely imaginary values of them, for which the fermion determinant and the path integral measure remain real and positive Then, under the same assumptions of analyticity leading to Eq. (1), and ensuring that the chosen set of imaginary chemical potentials stays within the analyticity domain, one can exploit analytic continuation to determine the nonlinear susceptibilities. In practice, one determines the generalized susceptibilities

$$
\chi_{i j k}=\sum_{\substack{l=i \\ m=\dot{j} \\ n=k}}^{\infty} \frac{\chi_{l m n}(0,0,0) \hat{\mu}_{u}^{l-i} \hat{\mu}_{d}^{m-j} \hat{\mu}_{s}^{n-k}}{(l-i) !(m-j) !(n-k) !}
$$

up to a given order $i+j+k$. From a global fit of their dependence on the imaginary chemical potentials $\hat{\mu}_{q}=$ $i \mu_{q, I} / T$ one can extract the higher-order susceptibilities.

This idea has been pursued in the past, both for the case of QCD with quark chemical potentials [16-24] and to determine the dependence of the free energy of pure gauge theories on the topological parameter $\theta$ [25-27]. Different strategies have been chosen in the various studies. For instance, in Refs. [22] and [24] only cumulants of order one have been measured [i.e. with $i+j+k=1$ in Eq. (3)], while up to fourth-order cumulants have been exploited in Ref. [23] (and also in Refs. [26,27] to study $\theta$ dependence); moreover, a two dimensional grid of imaginary chemical potentials has been used in Ref. [22] for $N_{f}=2$ QCD, while a one dimensional grid, corresponding to a variation of the baryon chemical potential $\mu_{B}$, has been exploited in the other cases.

The purpose of this study is to perform a systematic investigation of this technique for the case of $N_{f}=2+1$ QCD with physical quark masses, aimed both at identifying the optimal strategy in the choice of the simulation points and of the measured cumulants, and at testing its effectiveness. At the same time, we also aim at analyzing the possible systematic effects involved in the procedure, 
which are mostly related to the truncation of the series in Eq. (3) adopted in the fit.

To that purpose, we have performed a series of numerical simulations, mostly for $N_{t}=8$ lattices with an aspect ratio $L / T=4$, exploring temperatures in the range $135 \mathrm{MeV}<$ $T<350 \mathrm{MeV}$ while staying on a line of constant physics; simulations with different aspect ratios have been performed to estimate finite volume effects.

Numerical simulations have been performed for $O\left(10^{2}\right)$ different combinations of imaginary chemical potentials for each temperature and measuring cumulants up to order three.

In this way, we are able to determine the zero density quark number susceptibilities, with control over truncation effects, up to the sixth order for $T>T_{c}$ and up to the eighth order for $T<T_{c}$. A comparison with the standard method and with results obtained in previous studies is performed, in particular regarding the numerical efficiency of the strategy. In the low temperature region we also try to estimate the radius of convergence of the free energy expansion, in order to obtain information about the possible location of the second-order critical point at real $\mu_{B}$.

The paper is organized as follows. In Section II we present the lattice discretization, the strategy adopted for the choice of the simulation points (i.e. the different combinations of imaginary chemical potentials), and the expression of some of the computed observables. In Section III we present our results, and finally in Section IV we draw our conclusions.

\section{NUMERICAL METHODS AND SETUP}

We performed simulations of $N_{f}=2+1$ QCD in the presence of purely imaginary quark chemical potentials, $\mu_{f}=i \mu_{f, I}, \mu_{f, I} \in \mathbb{R}$, with $f=u, d, s$, considering the following Euclidean partition function of the discretized theory:

$$
\begin{aligned}
\mathcal{Z}= & \int \mathcal{D} U e^{-\mathcal{S}_{(\mathrm{sym})}} \prod_{f=u, d, s} \operatorname{det}\left(M_{\mathrm{st}}^{f}\left[U, \mu_{f, I}\right]\right)^{\frac{1}{4}} \\
\mathcal{S}_{(\mathrm{Sym})}= & -\frac{\beta}{3} \sum_{i, \mu \neq \nu}\left(\frac{5}{6} W_{i ; \mu \nu}^{1 \times 1}-\frac{1}{12} W_{i ; \mu \nu}^{1 \times 2}\right), \\
\left(M_{\mathrm{st}}^{f}\right)_{i, j}= & a m_{f} \delta_{i, j}+\sum_{\nu=1}^{4} \frac{\eta_{i ; \nu}}{2}\left[e^{i a \mu_{f, l} \delta_{\nu, 4}} U_{i ; \nu}^{(2)} \delta_{i, j-\hat{\nu}}\right. \\
& \left.-e^{-i a \mu_{f, I} \delta_{\nu, 4}} U_{i-\hat{\nu} ; \nu}^{(2) \dagger} \delta_{i, j+\hat{\nu}}\right] .
\end{aligned}
$$

$S_{(\text {Sym })}$ is the tree-level Symanzik action introduced in Refs. [28,29], with $W_{i ; \mu \nu}^{n \times m}$ being the trace of the $n \times m$ loop in the $(\mu, \nu)$ plane starting from site $i$. In order to reduce UV cutoff effects and taste symmetry violations, the staggered fermion matrix $M_{s t}^{f}$ is built up in terms of the twice stout-smeared links $U_{i ; \nu}^{(2)}$, which are constructed following the procedure described in Ref. [30] and using an isotropic smearing parameters $\rho=0.15$. As usual for finite $T$ simulations, periodic (antiperiodic) boundary conditions (b.c.) are taken for bosonic (fermionic) fields in the temporal direction, and periodic b.c. for all fields in the spatial directions.

For each flavor, we introduce the chemical potentials following the prescription of Ref. [31], i.e. by multiplying, in the fermion matrix, all the temporal links in the forward (backward) direction by $e^{+i a \mu_{f, I}}\left(e^{-i a \mu_{f, I}}\right)$ (see Refs. [32,33] for alternative discretizations). The chemical potentials coupled to quark number operators can be conveniently rewritten in terms of those coupled to the conserved charges, $B, Q$ and $S$, the relation being

$$
\begin{aligned}
& \mu_{u}=\mu_{B} / 3+2 \mu_{Q} / 3 \\
& \mu_{d}=\mu_{B} / 3-\mu_{Q} / 3 \\
& \mu_{s}=\mu_{B} / 3-\mu_{Q} / 3-\mu_{S} .
\end{aligned}
$$

As usual for staggered fermions simulations, the residual fourth degeneracy of the lattice Dirac operator is removed by the rooting procedure. The Rational Hybrid MonteCarlo algorithm [34-36] has been used to sample gauge configurations according to Eq. (4).

\section{A. Physical observables}

The observables measured during each simulation run correspond to the generalized susceptibilities $\chi_{i j k}(T, \mu)$ appearing in Eq. (3). In particular, we have considered all possible combinations with $i+j+k \leq 2$ for each simulation, and also the combinations with $i+j+k=3$ in some cases. Their explicit lattice version reads (we limit ourselves to the second order):

$$
\begin{aligned}
\chi_{1}^{f} \equiv & \frac{N_{t}}{4 N_{s}^{3}}\left\langle\operatorname{Tr}\left(\left(M_{s t}^{f}\right)^{-1} \frac{\partial M_{s t}^{f}}{\partial \mu_{f}}\right)\right\rangle \\
\chi_{2}^{f} \equiv & \frac{N_{t}}{N_{s}^{3}}\left(\frac{1}{4}\right)^{2}\left\langle\left[\operatorname{Tr}\left(\left(M_{s t}^{f}\right)^{-1} \frac{\partial M_{s t}^{f}}{\partial \hat{\mu}_{f}}\right)\right]^{2}\right\rangle \\
& -\frac{N_{t}}{N_{s}^{3}}\left(\frac{1}{4}\right)^{2}\left\langle\operatorname{Tr}\left(\left(M_{s t}^{f}\right)^{-1} \frac{\partial M_{s t}^{f}}{\partial \hat{\mu}_{f}}\right)\right\rangle^{2} \\
& +\frac{N_{t}}{4 N_{s}^{3}}\left\langle\operatorname{Tr}\left(\left(M_{s t}^{f}\right)^{-1} \frac{\partial^{2} M_{s t}^{f}}{\partial \hat{\mu}_{f}^{2}}\right)\right\rangle \\
& -\frac{N_{t}}{4 N_{s}^{3}}\left\langle\operatorname{Tr}\left(\left(M_{s t}^{f}\right)^{-1} \frac{\partial M_{s t}^{f}}{\partial \hat{\mu}_{f}}\left(M_{s t}^{f}\right)^{-1} \frac{\partial M_{s t}^{f}}{\partial \hat{\mu}_{f}}\right)\right\rangle \\
\chi_{2}^{i, j} \equiv & \frac{N_{t}}{N_{s}^{3}}\left(\frac{1}{4}\right)^{2}\left\langle\prod_{f=i, j}\left[\operatorname{Tr}\left(\left(M_{s t}^{f}\right)^{-1} \frac{\partial M_{s t}^{f}}{\partial \hat{\mu}_{f}}\right)\right]\right\rangle \\
& -\frac{N_{t}}{N_{s}^{3}}\left(\frac{1}{4}\right)^{2} \prod_{f=i, j}\left\langle\operatorname{Tr}\left(\left(M_{s t}^{f}\right)^{-1} \frac{\partial M_{s t}^{f}}{\partial \hat{\mu}_{f}}\right)\right\rangle
\end{aligned}
$$


where the presence of the factor $1 / 4$ is due to our staggered discretization. Their determination requires the evaluation of the following traces:

$$
\begin{gathered}
\operatorname{Tr}\left[\left(M_{s t}^{f}\right)^{-1} \frac{\partial M_{s t}^{f}}{\partial \mu_{f}}\right] \\
\operatorname{Tr}\left[\left(M_{s t}^{f}\right)^{-1} \frac{\partial M_{s t}^{f}}{\partial \mu_{f}}\right]^{2} \\
\operatorname{Tr}\left[\left(M_{s t}^{f}\right)^{-1} \frac{\partial^{(2)} M_{s t}^{f}}{\partial \mu_{f}^{2}}\right] .
\end{gathered}
$$

This is has been done, as usual, by means of noisy estimators, using 256 Gaussian random sources for each flavour. Confidence intervals and bias-subtraction for nonlinear estimators of susceptibilities have been performed by means of a Jackknife analysis [37].

\section{B. Choice of the simulation runs}

At fixed $N_{t}$, the temperature $T=1 /\left(N_{t} a\right)$ has been varied by tuning the bare coupling $\beta$ and the bare quark masses $m_{s}$ and $m_{u}=m_{d}=m_{l}$, so as to change the lattice spacing $a$ while staying on a line of constant physics, with $m_{\pi} \simeq 135 \mathrm{MeV}$ and $m_{s} / m_{l}=28.15$. This line has been determined by a spline interpolation of the determinations reported in Refs. [38-40].

For each temperature, the different combinations of imaginary quark chemical potentials have been chosen according to the following considerations. Our strategy is to obtain information about generalized susceptibilities at zero chemical potentials from the dependence on $\mu_{u}, \mu_{d}$ and $\mu_{s}$ of the measured lower-order susceptibilities described in the previous subsection. To that aim, in general we employ a truncated polynomial version of Eq. (3), i.e.

$$
\chi_{i j k}=\sum_{\substack{l=i \\ m=j \\ n=k}}^{l+m+n \leq p} \frac{\chi_{l m n}(0,0,0) \hat{\mu}_{u}^{l-i} \hat{\mu}_{d}^{m-j} \hat{\mu}_{s}^{n-k}}{(l-i) !(m-j) !(n-k) !}
$$

where the parameter $p$ fixes the maximum order we would like to determine.

The set of simulations points must contain values of $\mu_{u}$, $\mu_{d}$ and $\mu_{s}$ large enough, in order to be sensible to higherorder contributions. However, small values of the chemical potentials are important as well, in order to be able to check systematics related to truncation effects. Therefore a reasonable choice seems to take their values equally spaced between zero and some maximum reference value $\mu_{\max }$. This choice will be further discussed in Section III B.

In Ref. [22], a two-dimensional grid of equally spaced chemical potentials was considered for the case of $N_{f}=2$ QCD. In this case, considering a three-dimensional grid of equally spaced chemical potentials is surely not feasibile, since that would imply a number of different simulation runs scaling as $\mu_{\max }^{3}$ and reaching easily $O\left(10^{3}\right)$ for each temperature. Instead, we decided to fix the simulations points along well defined trajectories in the three-dimensional parameter space, in particular we did the following choices

$$
\begin{aligned}
& \left(\mu_{u}, \mu_{d}, \mu_{s}\right)=\left(i \mu_{I}, 0,0\right) \\
& \left(\mu_{u}, \mu_{d}, \mu_{s}\right)=\left(0,0, i \mu_{I}\right) \\
& \left(\mu_{u}, \mu_{d}, \mu_{s}\right)=\left(i \mu_{I}, i \mu_{I}, 0\right) \\
& \left(\mu_{u}, \mu_{d}, \mu_{s}\right)=\left(i \mu_{I},-i \mu_{I}, 0\right) \\
& \left(\mu_{u}, \mu_{d}, \mu_{s}\right)=\left(i \mu_{I}, i \mu_{I}, i \mu_{I}\right) \\
& \left(\mu_{u}, \mu_{d}, \mu_{s}\right)=\left(i \mu_{I},-i \mu_{I}, i \mu_{I}\right)
\end{aligned}
$$

where $\mu_{I}$ parametrizes each of the six different lines, with simulation points taken with a step size $\Delta \mu_{I}=0.025 \pi T$ between zero and a maximum value $\mu_{I, \max }$, which is the same for all the lines at a given temperature $T$. In this way, keeping the number of lines fixed, the computational effort scales linearly with $\mu_{I, \max }$.

Another aspect to be considered is whether the number of simulation points and measured observables is large enough to fix all generalized susceptibilities at a given order. Indeed, the number of independent generalized susceptibilities grows rapidly with the order: for $N_{f}=$ $2+1$ it is easy to prove, exploiting the symmetry $\chi_{\operatorname{lmn}}=$ $\chi_{m l n}$ due to the up and down quark mass degeneracy, that at order $N$ such number is given by $(N / 2+1)^{2}$. In general, the set of equations (12), which are used in the global fit, will involve only some linear combinations of such generalized susceptibilities, which depend on the number of lines in Eq. (13) and on the number of measured observables. Those linear combinations, for each order $N$ of generalized susceptibilities, define a matrix $A_{N}$, whose rank must be at least equal to $(N / 2+1)^{2}$ in order for the global fit to provide information about all independent susceptibilities.

In Fig. 1 we show the rank of $A_{N}$ in our setup [i.e. performing simulations along the six lines described in Eq. (13)], and assuming one measures all susceptibilities up to the second order, which has been our minimal choice for all temperatures. It is clear that our choice suffices to determine all the susceptibilities up to order eight, while only 33/36 generalized susceptibilities can be determined at order ten.

The situation can be improved (or worsened) by changing either the order of the measured susceptibilities or the number of lines where simulations are performed. For instance, in Figs. 2 and 3 we report the same information as in Fig. 1, respectively for the case in which only quark number densities are measured, and for the case in which the observables are extended up to susceptibilities of order three (keeping the number of lines fixed). Instead, in Fig. 4, we consider the case in which one still measures up to 


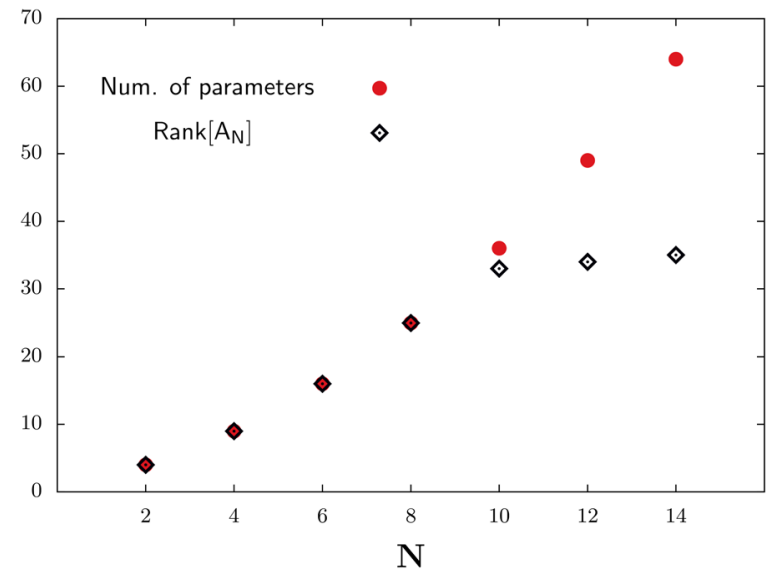

FIG. 1. Ranks of the $A_{N}$ matrices for some values of the order $N$, for simulation points chosen along the lines described in Eq. (13) and measuring as an input both quark number densities and second-order susceptibilities. Red circles correspond to the number of independent $\chi_{i j k}(T, \mu=0)$ at a given order.

second-order susceptibilities, but considers less or more lines of simulation points.

The naïve message would seem to measure more and more susceptibilities keeping the number of lines fixed, in order to avoid too many simulations. However, as we have already discussed, the precision on the observables degrades rapidly with the order, so what is the optimal strategy is nontrivial and will be discussed in the following, based on numerical results.

There is no special limitation on the choice of $\mu_{I, \max }$ in the low temperature regime, $T<T_{c}$, where the partition function is an analytic function in all chemical potentials. In the high- $T$ region, instead, the range of available chemical potentials is limited by the presence of Roberge-Weiss (RW) or RW-like phase transitions, associated to a sudden change of the expectation value of the Polyakov loop, related to different realization of the center symmetry breaking. The genuine RW transition [41] is met when

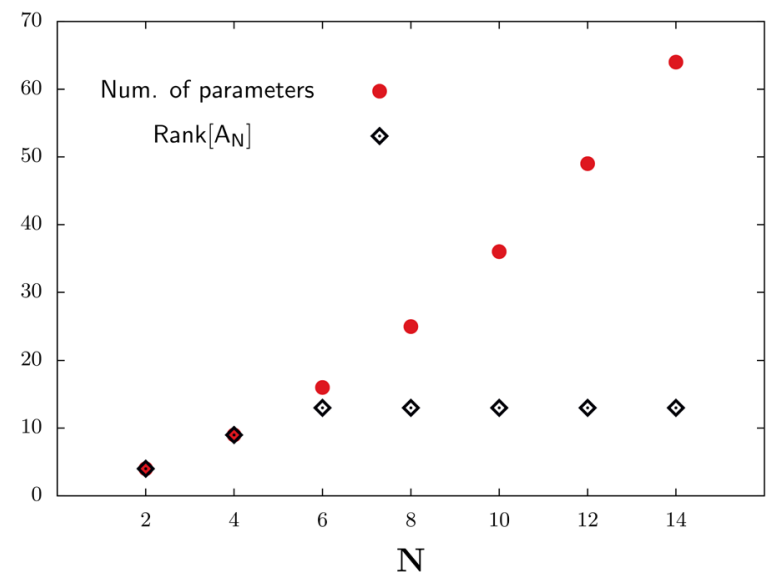

FIG. 2. Same as in Fig. 1, but with just quark number densities taken as an input.

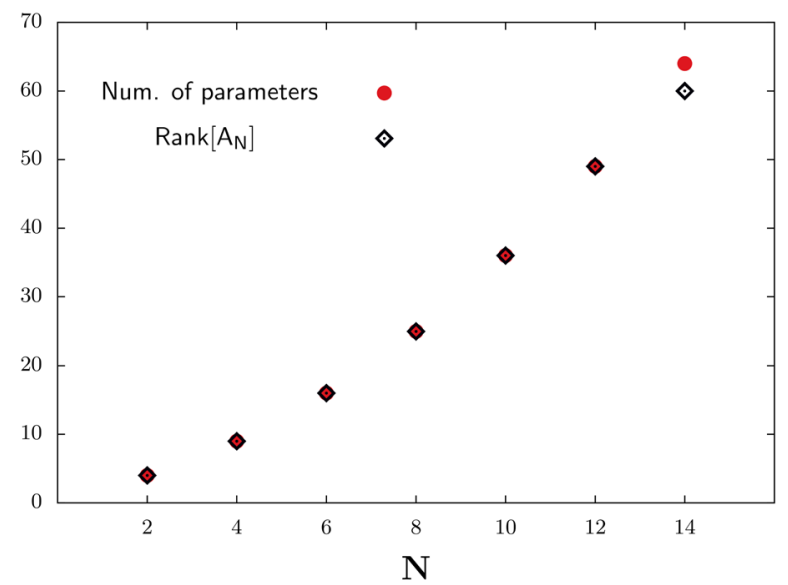

FIG. 3. Same as in Fig. 1, apart from the fact that also quark number susceptibilities of order three are measured and added as an input.

moving along the line $\mu_{u}=\mu_{d}=\mu_{s}=i \mu_{I}$, which corresponds to a pure baryon chemical potential $\mu_{B}=3 i \mu_{I}$ [i.e. $\mu_{Q}=\mu_{S}=0$, see Eq. (7)]: a purely imaginary $\mu_{B}$ corresponds to a global rotation of the temporal boundary conditions for fermion fields, leading to a rotation of the fermion contribution to the effective potential of the Polyakov loop and to a sudden change of the global minimum for

$$
\mu_{I} / T=(2 n+1) \pi / 3
$$

where $n$ is a relative integer. The phase diagram in the $T-\mu_{I}$ looks as in Fig. 5: the vertical lines are first-order RW transition lines, they start from a critical temperature $T_{R W}>T_{c}$, whose value is about $200 \mathrm{MeV}$ for $N_{t}=8$ and about $208 \mathrm{MeV}$ in the continuum limit [42]. The first RW

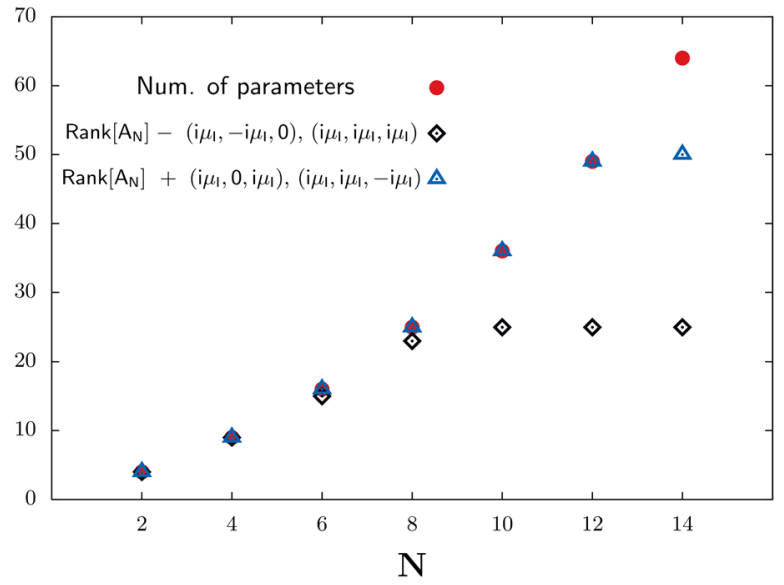

FIG. 4. Same as in Fig. 1, but considering less or more lines of simulation points. Triangles correspond to the case in which two additional lines, $\left(i \mu_{I}, 0, i \mu_{I}\right)$ and $\left(i \mu_{I}, i \mu_{I},-i \mu_{I}\right)$, have been added, while diamonds to the case in which two lines, $\left(i \mu_{I},-i \mu_{I}, 0\right)$ and $\left(i \mu_{I}, i \mu_{I}, i \mu_{I}\right)$, are not considered. 


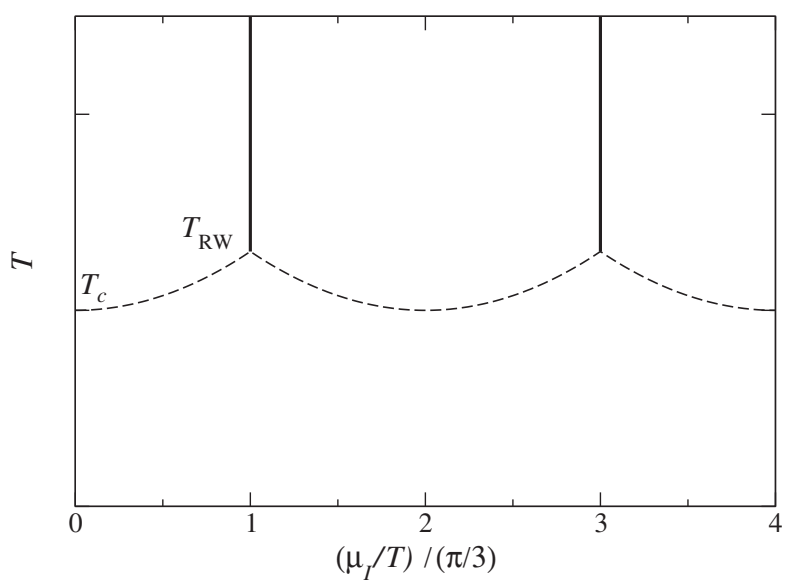

FIG. 5. Sketch of the phase diagram in the $T-\mu_{I}$ plane. In this case $\left(\mu_{u, d, s}=i \mu_{I}\right)$ RW lines are exactly vertical and located at $\mu_{I} / T=(2 n+1) \pi / 3$. Solid lines represent first-order phase transitions separating sectors with different orientation of the Polyakov loop while dashed lines correspond to the analytic continuation of the pseudocritical line.

line limits the region which is analytically connected to the points at zero chemical potentials, hence one has to take $\mu_{I, \max } / T<\pi / 3$ for $T>T_{R W}$.

For intermediate temperatures, $T_{c}<T<T_{R W}$, no critical points are expected, since the analytic continuation of the chiral transition line (dashed curve in Fig. 5) is only pseudocritical. However, one expects that, when crossing this pseudocritical line, the dependence of the free energy $F$ on the chemical potentials may become less smooth, so that systematic effects due to truncation may become more severe, resulting in an effective limitation of the explorable range of $\mu$.

Similar dynamics take place along the other lines, i.e. in the more general case $\mu_{Q}, \mu_{S} \neq 0$ : in those cases the exact position of the first RW-like line depends on the quark masses and on $T$; however, one can safely state that it will occur for $\mu_{I} / T>\pi / 3$, since in this case the different flavors tend to orient the Polyakov loop along different directions in the complex plane (see Refs. $[43,44]$ for a more detailed discussion about this point). For instance, along the line $\mu_{u}=\mu_{d}=i \mu_{I}$ and $\mu_{s}=0$, it occurs for $\mu_{I} / T \sim 0.45 \pi$ [44].

Following the discussion above, we have taken $\mu_{I, \max } / T<\pi / 3$ for all temperatures above $T_{c}$. Around $T_{c}$, the range of chemical potentials actually used in the global fit will be decided on the basis of the quality and of the stability of the fit itself.

\section{NUMERICAL RESULTS}

Most simulations have been performed on a $32^{3} \times 8$ lattice for various temperatures: the complete list, including the values of the bare parameters, is reported in Table I. For each run, 1500 trajectories of unitary length have been
TABLE I. List of simulated temperatures and associated values of $\beta, m_{l}, m_{s} ; \mu_{I, \max }$ represents the maximum value of the imaginary chemical potential used in the simulations. The value of $T$ is affected by an uncertainty related to the determination of the physical scale, which for the discretization adopted in our study is of the order of $2 \%-3 \%$ [38-40].

\begin{tabular}{llllc}
\hline \hline$T[\mathrm{MeV}]$ & \multicolumn{1}{c}{$\beta$} & \multicolumn{1}{c}{$m_{l}$} & \multicolumn{1}{c}{$m_{s}$} & $\mu_{I, \max } / T$ \\
\hline 135 & 3.61 & 0.002831 & 0.07971 & $0.8 \pi$ \\
143 & 3.63 & 0.002621 & 0.07378 & $0.8 \pi$ \\
149 & 3.645 & 0.002479 & 0.06978 & $0.4 \pi$ \\
155 & 3.66 & 0.002350 & 0.06614 & $0.4 \pi$ \\
160 & 3.67 & 0.002270 & 0.0639 & $0.3 \pi$ \\
170 & 3.69 & 0.002126 & 0.5984 & $0.3 \pi$ \\
200 & 3.755 & 0.001763 & 0.04963 & $0.3 \pi$ \\
230 & 3.815 & 0.001516 & 0.04267 & $0.3 \pi$ \\
260 & 3.87 & 0.001341 & 0.03775 & $0.3 \pi$ \\
300 & 3.94 & 0.001168 & 0.3287 & $0.3 \pi$ \\
350 & 4.0225 & 0.0009920 & 0.2792 & $0.3 \pi$ \\
\hline \hline
\end{tabular}

performed. We measured susceptibilities on configurations separated by 10 trajectories to reduce autocorrelation effects. ${ }^{1}$ A few additional simulations have been performed on lattices with different aspect ratios, both below and above $T_{c}$, to check for finite size effects.

As outlined above, our strategy, for each temperature, has been to perform a global fit, according to Eq. (12), of the dependence on the chemical potentials of all quark number densities and susceptibilities up to order two, along the trajectories in the three-dimensional chemical potential space described in Eq. (13). A subsample of such global fit is reported in Fig. 6 for $T=149 \mathrm{MeV}$, where we show some of the best fit polynomials obtained according to Eq. (12) with a truncation $p=8$.

\section{A. Analysis of systematic errors}

The main source of systematic error, in the analytic continuation method, comes from the ambiguity in the choice of the fitting function. In our case this means that coefficients resulting from the global fit procedure, i.e. the generalized susceptibilities $\chi_{i j k}(T)$, may depend on the order of the polynomial (i.e. on the truncation order) as well as on the fitting range.

As a general procedure to keep this systematic error under control, we started with ranges of $\mu_{I}$, going from zero up to a maximum value $\bar{\mu}_{I}$, small enough so that a lowestorder polynomial could provide a good description of the data. Next, we increased the upper value of the range, $\bar{\mu}_{I}$, keeping the polynomial degree fixed, as long as reasonable

\footnotetext{
${ }^{1}$ To check that our choice was reasonable, we measured the autocorrelation times of some typical observables. Throughout the explored range of temperatures, the autocorrelation times of the plaquette and of the quark number densities is 4-6 trajectories, whereas it is of $\mathrm{O}(10)$ trajectories for the chiral condensate.
} 

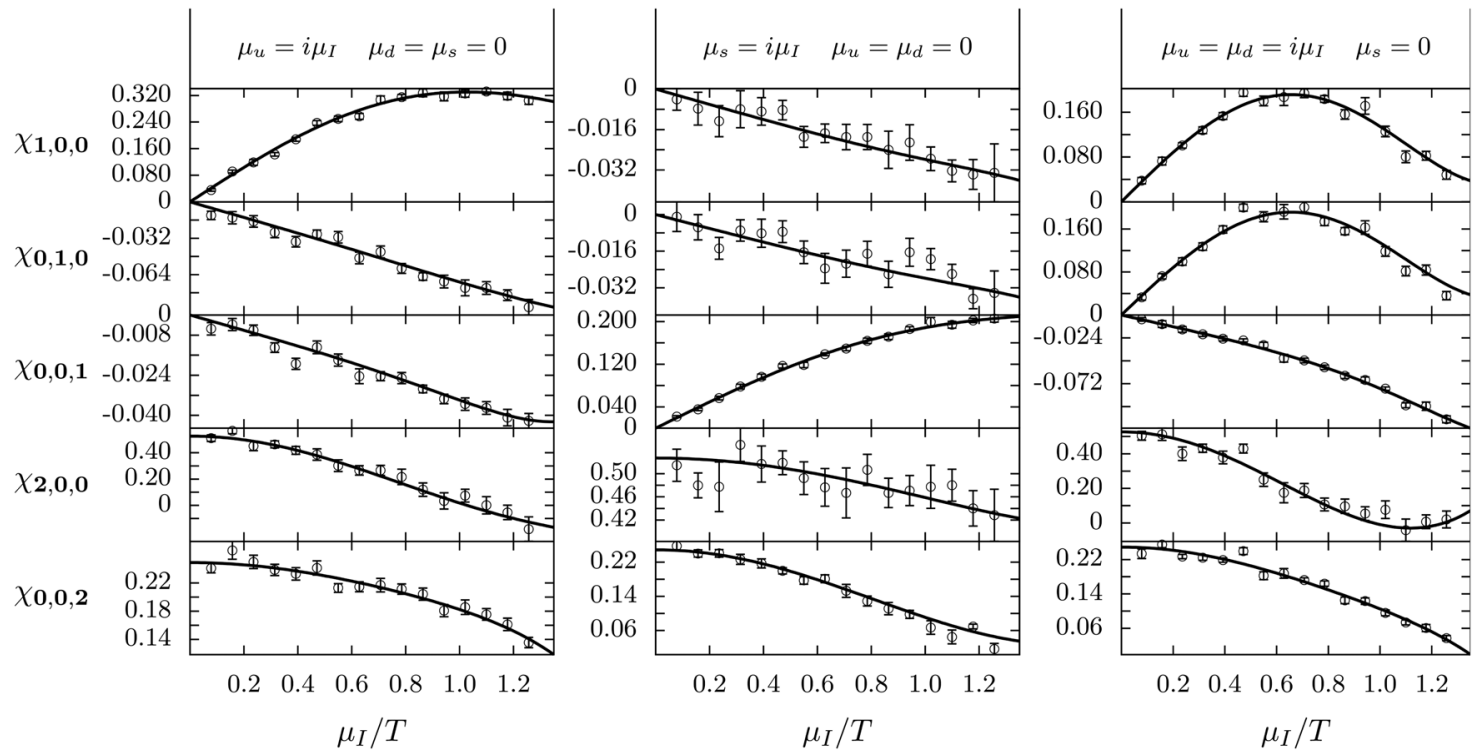

FIG. 6. Example of the global fit for $T=149 \mathrm{MeV}$. We show only a subsample of a total of 54 polynomial fits which are performed at the same time (three densities plus six second-order susceptibilities fitted along six different trajectories). The best-fit functions are taken according to Eq. (12) with a truncation to order eight. The reduced $\tilde{\chi}^{2}$ is 1.3 . Notice that in the global fit we did not take into account crosscorrelations between susceptibilities measured at the same chemical potential, hence the covariance matrix has a simple diagonal form.

values of reduced chi-squared test, $\tilde{\chi}^{2}$, were obtained. Otherwise, the polynomial order was increased in order to go back to $\tilde{\chi}^{2} \simeq 1$ : at this stage, the stability of the previously determined coefficients was checked, and any variation going beyond the statistical errors (obtained in the

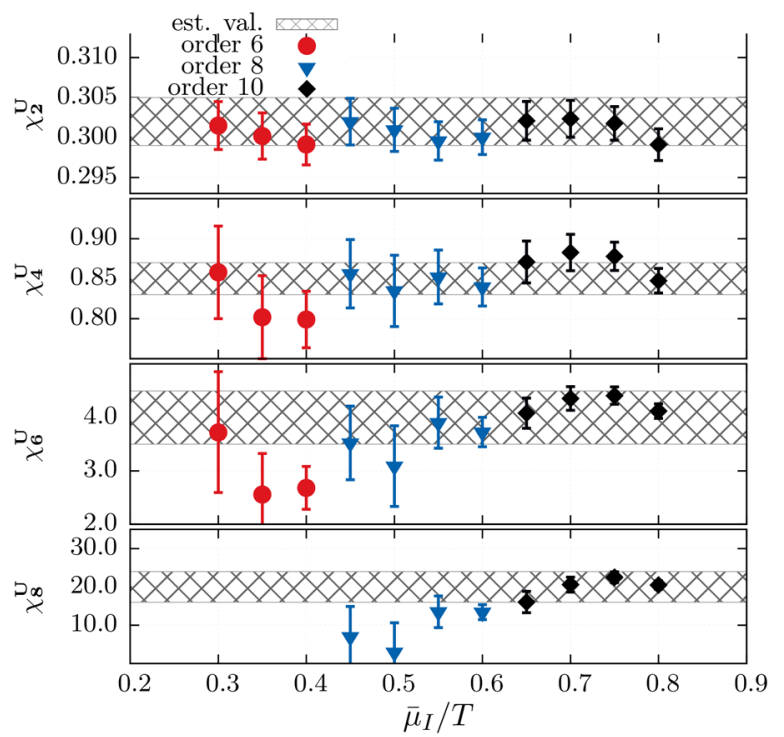

FIG. 7. An example of the procedure followed to determine systematic errors. The errorbars represent the statistical error obtained in the global best fit. The polynomial degree is increased every time that the global best fit yields nonacceptable values of the reduced chi-squared $\tilde{\chi}^{2}$. Circles, triangles and diamonds refer to a global fit performed with a polynomial of order $6,8,10$, respectively, while the grey bands represent the final estimate. Data refer to simulations on the $32^{3} \times 8$ lattice at $T=135 \mathrm{MeV}$. global best-fit procedure) was added as a systematic error to the final determination.

An example of this procedure is reported in Fig. 7, where we show the evolution of some susceptibilities as the fit range or the polynomial order is changed, for $T=135$.

To investigate finite size effects, we carried out simulations on $N_{t}=6$ lattices for $T=170 \mathrm{MeV}$ and on $N_{t}=8$ lattices at $T=350 \mathrm{MeV}$, considering three different values for the spatial volume, $N_{s}=16,20,24$ for the $N_{t}=6$ lattice and $N_{s}=24,32,40$ for the $N_{t}=8$ one. In Figs. 8 and 9 our results for the up-quark and up-strange susceptibilities are shown. The analysis indicates that no finite volume effects are visible, within our present statistical accuracy, when passing from aspect ratio 4 to aspect ratio 3.3 for $T=170 \mathrm{MeV}$, and from aspect ratio 5 to aspect ratio 4 at $350 \mathrm{MeV}$. It is interesting to notice a reduction of

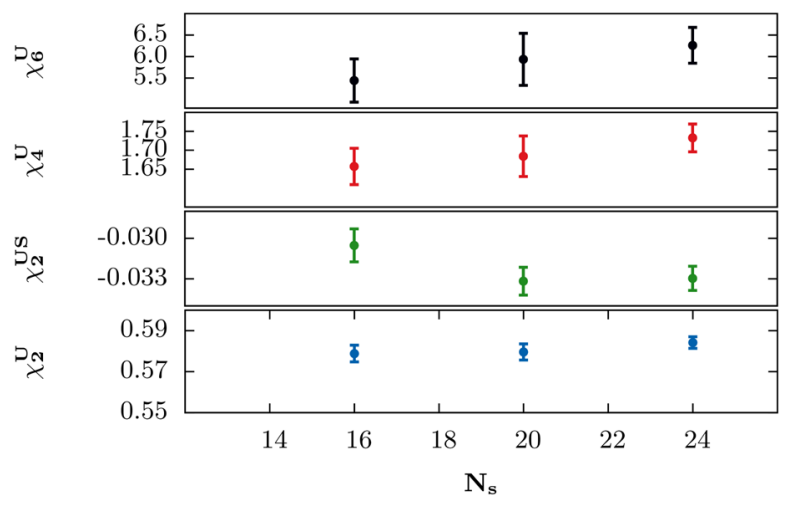

FIG. 8. Variation of some quark susceptibilities with the volume size at $T=170 \mathrm{MeV}$ on the $N_{t}=6$ lattice. 


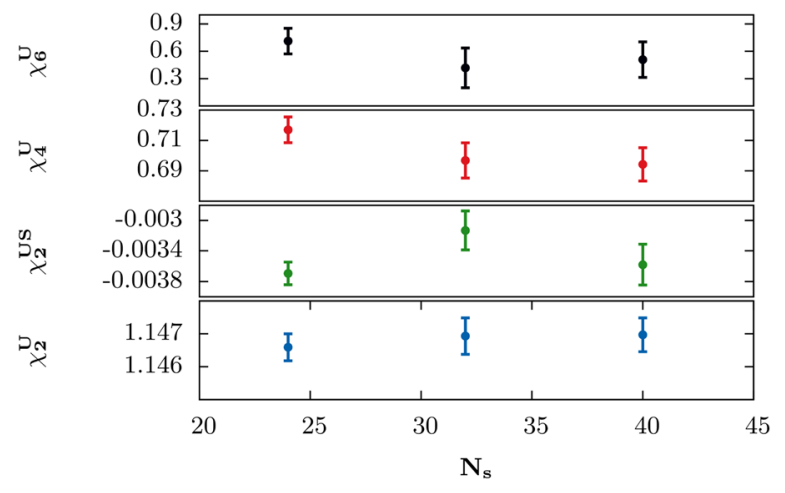

FIG. 9. Same as in Fig. 8 for $T=350 \mathrm{MeV}$ on the $N_{t}=8$ lattice.

the statistical error on the larger lattices: since the same statistics have been adopted for the different spatial sizes, this can be related to the fact that some of the fitted observables (quark number densities) are self-averaging, i.e. their statistical fluctuations decreases as $1 / \sqrt{V}$, while the other are characterized by statistical fluctuations which are independent of $V$ (the second-order susceptibilities), so that, on the whole, one expects some gain in accuracy when moving to larger volumes.

This is visible even for the case of the sixth-order susceptibilities, whereas in the direct computation at $\mu=0$ their determination would be affected by a relative error growing like $\propto V^{2}$.

\section{B. Efficiency of the method and comparison with a direct determination at $\mu=0$}

At this stage we are in a position to discuss the efficiency of the method, i.e. to compare the total computational effort in the direct calculation and in the analytic continuation method. In Table II, we compare results obtained for

TABLE II. Comparison of results obtained for second- and fourth-order susceptibilities from the global fit procedure, with the ones obtained from the standard computation and a statistics similar to that accumulated for $O(10)$ simulation points at imaginary $\mu$. The total computational effort spent in the global fit is larger than that spent in the standard case by a factor 10 for $T=143 \mathrm{MeV}$, and 3 for $T=260 \mathrm{MeV}$.

\begin{tabular}{lccccc}
\hline \hline & \multicolumn{2}{c}{$\mu=0$} & & \multicolumn{2}{c}{ From global fit } \\
\cline { 2 - 3 } \cline { 5 - 5 }$T[\mathrm{MeV}]$ & 143 & 260 & & 143 & 260 \\
\hline$\chi_{2,0,0}$ & $0.410(12)$ & $1.0880(12)$ & $0.4160(40)$ & $1.0883(8)$ \\
$\chi_{0,0,2}$ & $0.1862(24)$ & $1.0250(13)$ & & $0.1865(15)$ & $1.0255(10)$ \\
$\chi_{1,0,1}$ & $-0.031(3)$ & $-0.00774(57)$ & $-0.031(1)$ & $-0.00740(40)$ \\
$\chi_{1,1,0}$ & $-0.075(8)$ & $-0.0091(6)$ & $-0.0680(20)$ & $-0.0080(5)$ \\
$\chi_{4,0,0}$ & $1.1(8)$ & $0.65(1)$ & & $1.250(70)$ & $0.635(20)$ \\
$\chi_{0,0,4}$ & $0.336(40)$ & $0.721(15)$ & & $0.300(15)$ & $0.710(30)$ \\
$\chi_{2,0,2}$ & $0.17(7)$ & $0.0452(35)$ & $0.1195(33)$ & $0.0440(60)$ \\
$\chi_{2,2,0}$ & $0.2(3)$ & $0.043(4)$ & $0.2924(82)$ & $0.038(5)$ \\
\hline \hline
\end{tabular}

second and fourth-order susceptibilities, for two values of the temperature $(T=143,260 \mathrm{MeV})$, from the standard method and from the global fit, in order to test the efficiency of our method both in the confined and in the plasma phase.

In order to make a proper comparison, one must take the relative computational effort into account. In both cases, each measurement involved 256 random sources; however, five matrix inversions for each flavor were used in the standard determination, in order to obtain all susceptibilities up to order four, and just two inversions in the analytic continuation case, in order to obtain all the second-order susceptibilities involved in the global fit. For the standard determination, we performed measurements on 1000 configurations for $T=143 \mathrm{MeV}$ and 2000 configurations for $T=260 \mathrm{MeV}$, each separated by 10 RHMC trajectories; the relative $\operatorname{cost}^{2}$ of each measurement compared to each MD trajectory was about 40 for $T=143 \mathrm{MeV}$ and about 7 for $T=260 \mathrm{MeV}$. The determination from analytic continuation, considering all simulation points, involved measurements on $20 \mathrm{~K}$ configurations for $T=143 \mathrm{MeV}$ and $7 \mathrm{~K}$ configurations for $T=260 \mathrm{MeV}$, each separated by 10 RHMC trajectories; the relative cost of each measurement compared to each MD trajectory was about 16 for $T=143 \mathrm{MeV}$ and about 3 for $T=260 \mathrm{MeV}$. Summing up, we can estimate a total computational effort spent in the global fit which is larger than that spent in the standard case, by a factor 10 for $T=143 \mathrm{MeV}$, and 3 for $T=260 \mathrm{MeV}$. In standard importance sampling, error bars scale according to the inverse square root of the sample size; therefore, rescaling appropriately the error, we can compare the two determination at fixed machine time.

A clear result, emerging from Table II, is that the standard method is comparable, or even more efficient than analytic continuation in the deconfined phase, for all susceptibilities up to order four. For $T=143 \mathrm{MeV}$, i.e. below the pseudocritical temperature, the situation is quite different. Analytic continuation has still an efficiency comparable to the standard method for second-order susceptibilities; however, for fourth-order susceptibilities, the improvement is dramatic: analytic continuation leads to an improvement which is of order 10, in terms of time machine, for the diagonal light quark susceptibility, $\chi_{4,0,0}$, and grows up to order 100 for the nondiagonal susceptibilities (no significant improvement is observed, instead, for $\left.\chi_{0,0,4}\right)$.

For sake of completeness, in Table III, we report the values of some second- and fourth-order susceptibilities as a function of the number of random sources. Our determinations suggest that the error over fourth-order cumulants decreases more sharply with respect to the

\footnotetext{
${ }^{2}$ This estimate is specific to our code implementation on the BlueGene/Q machine and could be different for other implementations or machines.
} 
TABLE III. A subset of second- and fourth-order susceptibilities as a function of the number of random sources is shown. Data refer to simulations at $\mu=0$ and $T=143 \mathrm{MeV}$.

\begin{tabular}{lcccc}
\hline \hline$n_{\text {copies }}$ & 64 & 128 & 256 & 512 \\
\hline$\chi_{2,0,0}$ & $0.401(37)$ & $0.400(19)$ & $0.410(12)$ & $0.4158(81)$ \\
$\chi_{0,0,2}$ & $0.186(5)$ & $0.191(3)$ & $0.1862(24)$ & $/ /$ \\
$\chi_{1,0,1}$ & $-0.031(9)$ & $-0.026(5)$ & $-0.031(3)$ & $/ /$ \\
$\chi_{1,1,0}$ & $-0.084(24)$ & $-0.084(12)$ & $-0.075(8)$ & $/ /$ \\
$\chi_{4,0,0}$ & $9(7)$ & $3(2)$ & $1.1(8)$ & $1.05(35)$ \\
$\chi_{0,0,4}$ & $0.16(19)$ & $0.33(6)$ & $0.336(40)$ & $/ /$ \\
$\chi_{2,0,2}$ & $0.12(45)$ & $0.10(15)$ & $0.17(7)$ & $/ /$ \\
$\chi_{2,2,0}$ & $3(3)$ & $0.7(9)$ & $0.2(3)$ & $/ /$ \\
\hline \hline
\end{tabular}

second-order ones when increasing the number of random vectors. This different behaviour is expected, since fourthorder cumulants are composed by terms which involve products of three and four traces and their uncertainty decreases more sharply as the number of random vectors is increased. For sure, by increasing sufficiently the number of random sources error saturation will occur due to the fact that gauge fluctuations dominate over random noise. However, it is possible that going from 256 to 512 or 1024 random sources, this trend continues to be valid. Therefore, uncertainties over quark number susceptibilities determined from direct sampling and from the global fit could scale differently as the number of sources is increased, leading to a slight change in the efficiency comparison. This, however, should not change the main conclusion, i.e. that analytic continuation gains a large factor, below $T_{c}$, starting from fourth-order susceptibilities, and especially for mixed ones.

Let us try to give a few possible explanations for the fact that analytic continuation seems to be not so convenient above $T_{c}$. A significant role is surely played by the fact that small eigenvalues of the Dirac operator are strongly suppressed above $T_{c}$ (due to chiral symmetry restoration), so that, at the same time, the multiple inversions needed in the standard method are less costly, and fluctuations in the noisy estimators are suppressed; this effect is visible even below $T_{c}$, for susceptibilities involving strange quarks, which have a larger mass, for which the gain of analytic continuation is less marked. Another possible factor is related to the fact that we are working with a fixed aspect ratio, so that simulations at higher temperatures are based on smaller physical volumes, where problems related to the lack of self-averaging are expected to be less severe.
Finally, in the high temperature phase analytic continuation is surely disfavored by the reduced range of explorable chemical potentials, due to RW or RW-like transitions: that affects both the statistical accuracy of the global fit and, even more important, the systematic uncertainty related to truncation effects.

Another question that we would like to answer, which regards the optimal strategy to be followed, is whether there is any significant gain in trying measuring also susceptibilities of order larger than 2 . In other case, like in the use of analytic continuation for the study of $\theta$ dependence [25-27], the issue is not very important, since one can compute cumulants of the topological charge at any chosen order with no significant computational overhead; in this case instead, going one order further in the measure of cumulants means adding new inversions of the Dirac operator, with a considerable overhead. To this purpose, we performed trial simulations at $T=143 \mathrm{MeV}$, measuring all quark number susceptibilities up to order three, and observing how errors change as a function of the order of the susceptibilities included in the global fit. Some results are reported in Fig. 11. A remarkable improvement is achieved when adding second-order susceptibilities to the information coming from just quark number densities: the improvement reaches up to a factor 3 , in terms of error reduction. On the other hand, including the third order as well has a low impact since, in general, only a little gain is achieved.

Finally, we would like to discuss whether the choice of equally distributed simulation points, along the imaginary chemical potential axes, is optimal or not. In principle, one would expect that having more simulations where cumulants get larger contributions from higher-order terms of the expansion, i.e. at larger values of $\mu_{I}$, would be better, in order to obtain more information on higher-order susceptibilities. However, one must consider that, in order to properly perform the analysis on the systematic error related to the series truncation, which has been illustrated in the previous subsection, one needs enough determinations at small $\mu_{I}$ as well. In fact, we have tried to perform the analysis on various subsets of our simulation points, keeping more data either in the high or in the low $\mu_{I}$ region and comparing the final error in the various cases, after normalizing it to the total computational effort needed. The result is that there is indeed a benefit in having more points in the high $\mu_{I}$ region when one considers just the statistical error; however, that disappears when the total error

TABLE IV. Table of second-order susceptibilities obtained from polynomial fits. Errors are calculated taking into account both statistical uncertainties and systematic effects.

\begin{tabular}{|c|c|c|c|c|c|c|c|c|c|c|c|}
\hline$T[\mathrm{MeV}]$ & 135 & 143 & 149 & 155 & 160 & 170 & 200 & 230 & 260 & 300 & 350 \\
\hline$\chi_{0,0,2}$ & $0.12770(90)$ & $0.1865(15)$ & $0.2485(10)$ & $0.3230(20)$ & $0.3800(20)$ & $0.4980(30)$ & $0.7960(20)$ & $0.9485(15)$ & $1.0255(10)$ & $1.0815(10)$ & $1.12250(70)$ \\
\hline$\chi_{1,0,1}$ & $-0.02820(50)$ & $-0.031(1)$ & $-0.03050(70)$ & $-0.02780(80)$ & $-0.0285(10)$ & $-0.0251(11)$ & $-0.01520(70)$ & $-0.00960(40)$ & $-0.00740(40)$ & $-0.00500(40)$ & $-0.00320(30)$ \\
\hline$\chi_{1,1,0}$ & $-0.0698(15)$ & $-0.0680(20)$ & $-0.0687(18)$ & $-0.0566(16)$ & $-0.0540(25)$ & $-0.0462(20)$ & $-0.0187(10)$ & $-0.01130(60)$ & $-0.00800(50)$ & $-0.00510(30)$ & $-0.00343(35)$ \\
\hline$\chi_{2,0,0}$ & $0.3020(30)$ & $0.4160(40)$ & $0.5275(25)$ & $0.6480(40)$ & $0.7080(40)$ & $0.8170(60)$ & $0.9888(15)$ & $1.0515(12)$ & $1.08830(80)$ & $1.12200(60)$ & $1.14700(60)$ \\
\hline
\end{tabular}


TABLE V. Same as in Table IV for the fourth-order susceptibilities.

\begin{tabular}{|c|c|c|c|c|c|c|c|c|c|c|c|}
\hline$T[\mathrm{MeV}]$ & 135 & 143 & 149 & 155 & 160 & 170 & 200 & 230 & 260 & 300 & 350 \\
\hline$\chi_{0,0,4}$ & $0.195(10)$ & $0.300(15)$ & $0.411(13)$ & $0.470(30)$ & $0.67(10)$ & $0.72(12)$ & $0.810(80)$ & $0.75(5)$ & $0.710(30)$ & $0.640(35)$ & $0.690(30)$ \\
\hline$\chi_{1,0,3}$ & $-0.0266(32)$ & $-0.0188(60)$ & $-0.0200(70)$ & $0.0060(80)$ & $0.000(40)$ & $0.038(24)$ & $-0.023(25)$ & $0.010(10)$ & $0.007(10)$ & $0.0020(60)$ & $0.0080(70)$ \\
\hline$\chi_{1,1,2}$ & $0.0080(20)$ & $0.010(4)$ & $0.02(1)$ & $0.0210(60)$ & $0.013(15)$ & $0.010(15)$ & $0.025(25)$ & $0.0022(64)$ & $0.0000(50)$ & $-0.0065(45)$ & $-0.0020(40)$ \\
\hline$\chi_{2,0,2}$ & $0.0790(25)$ & $0.1195(33)$ & $0.160(10)$ & $0.180(20)$ & $0.177(20)$ & $0.150(20)$ & $0.084(20)$ & $0.025(10)$ & $0.0440(60)$ & $0.0330(50)$ & $0.0250(50)$ \\
\hline$\chi_{2,1,1}$ & $0.0084(20)$ & $0.0090(20)$ & $0.0075(75)$ & $0.021(8)$ & $0.010(8)$ & $0.0028(83)$ & $0.000(10)$ & $0.0140(40)$ & $-0.0020(30)$ & $0.0012(16)$ & $0.0030(30)$ \\
\hline$\chi_{2,2,0}$ & $0.2115(80)$ & $0.2924(82)$ & $0.364(12)$ & $0.430(20)$ & $0.310(35)$ & $0.140(40)$ & $0.081(14)$ & $0.0434(66)$ & $0.0380(50)$ & $0.0280(45)$ & $0.026(8)$ \\
\hline$\chi_{3,0,1}$ & $-0.0060(40)$ & $0.010(10)$ & $0.0208(73)$ & $0.050(15)$ & $-0.01(3)$ & $0.000(25)$ & $0.020(20)$ & $0.0080(70)$ & $0.0040(60)$ & $0.0037(51)$ & $0.0050(50)$ \\
\hline$\chi_{3,1,0}$ & $-0.0160(45)$ & $0.010(9)$ & $0.0230(90)$ & $0.081(15)$ & $0.004(45)$ & $-0.035(25)$ & $0.005(15)$ & $0.010(10)$ & $0.0064(54)$ & $0.0018(28)$ & $-0.0009(38)$ \\
\hline$\chi_{4,0,0}$ & $0.850(20)$ & $1.250(70)$ & $1.410(40)$ & $1.55(15)$ & $1.30(20)$ & $0.840(70)$ & $0.620(60)$ & $0.590(30)$ & $0.635(20)$ & $0.700(20)$ & $0.710(25)$ \\
\hline
\end{tabular}

TABLE VI. Same as in Table IV for the sixth-order susceptibilities.

\begin{tabular}{|c|c|c|c|c|c|c|c|c|c|c|c|}
\hline$T[\mathrm{MeV}]$ & 135 & 143 & 149 & 155 & 160 & 170 & 200 & 230 & 260 & 300 & 350 \\
\hline & $0.60(15)$ & $0.75(10)$ & $1.00(50)$ & $0.69(18)$ & $1.98(87)$ & $1.56(98)$ & $-1.1(21)$ & $0.43(56)$ & $0.29(36)$ & $-1.25(75)$ & $-0.10(33)$ \\
\hline$\chi_{1,0,5}$ & $-0.017(17)$ & $0.080(30)$ & $-0.060(90)$ & $0.30(35)$ & $-0.19(43)$ & $0.63(50)$ & $-1.00(70)$ & $0.09(17)$ & $0.06(11)$ & $0.01(11)$ & $0.043(87)$ \\
\hline$\chi_{1,1,4}$ & $-0.005(15)$ & $-0.003(12)$ & $0.049(42)$ & $0.045(60)$ & $0.00(23)$ & $0.06(32)$ & $0.5(10)$ & $-0.067(92)$ & $-0.018(60)$ & $-0.133(67)$ & $-0.035(44)$ \\
\hline$\chi_{2,0,4}$ & $0.080(20)$ & $0.142(21)$ & $0.25(12)$ & $0.16(10)$ & $0.11(24)$ & $-0.14(38)$ & $0.43(60)$ & $-0.25(35)$ & $-0.076(64)$ & $-0.190(82)$ & $-0.020(48)$ \\
\hline$\chi_{2,1,3}$ & $0.010(10)$ & $0.0112(90)$ & $-0.10(10)$ & $0.019(26)$ & $0.09(13)$ & $-0.20(36)$ & $-0.50(23)$ & $0.05(15)$ & $-0.007(31)$ & $0.052(33)$ & $0.030(22)$ \\
\hline $\begin{array}{l}\lambda_{2,1,3} \\
\chi_{2,2,2}\end{array}$ & $0.0050(50)$ & $0.0200(80)$ & $0.000(70)$ & $-0.05(10)$ & $0.10(20)$ & $-0.110(70)$ & $0.02(14)$ & $-0.032(34)$ & $-0.010(19)$ & $-0.044(25)$ & $-0.015(13)$ \\
\hline$\chi_{3,0,3}$ & $-0.0160(60)$ & $0.023(13)$ & $-0.002(15)$ & $0.005(20)$ & $0.082(42)$ & $-0.29(23)$ & $1.7(1.0)$ & $0.70(70)$ & $0.001(97)$ & $0.012(43)$ & $0.085(68)$ \\
\hline$\chi_{3,1,2}$ & $0.0100(50)$ & $0.0070(80)$ & $0.050(50)$ & $-0.005(13)$ & $-0.037(53)$ & $-0.062(69)$ & $0.03(10)$ & $-0.015(26)$ & $0.019(16)$ & $-0.030(19)$ & $-0.011(12)$ \\
\hline$\chi_{3,2,1}$ & $0.010(10)$ & $-0.002(20)$ & $0.000(13)$ & $0.035(25)$ & $-0.180(50)$ & $-0.130(50)$ & $0.027(58)$ & $0.018(18)$ & $-0.017(12)$ & $0.009(10)$ & $0.0006(81)$ \\
\hline$\chi_{3,3,0}$ & $0.097(50)$ & $0.035(40)$ & $0.11(10)$ & $-0.46(17)$ & $-0.53(18)$ & $-0.10(14)$ & $0.050(70)$ & $-0.001(23)$ & $0.020(23)$ & $0.015(16)$ & $0.0006(81)$ \\
\hline$\chi_{4,0,2}$ & $0.127(21)$ & $0.190(40)$ & $0.30(15)$ & $0.230(50)$ & $-0.21(27)$ & $-0.46(31)$ & $-0.32(51)$ & $-0.23(12)$ & $-0.058(52)$ & $0.120(83)$ & $-0.041(36)$ \\
\hline$\chi_{4,1,1}$ & $0.010(10)$ & $0.000(15)$ & $-0.008(16)$ & $-0.003(21)$ & $0.040(87)$ & $-0.083(93)$ & $0.08(10)$ & $-0.00(10)$ & $-0.013(17)$ & $-0.005(16)$ & $0.014(13)$ \\
\hline$\chi_{4,2,0}$ & $0.325(50)$ & $0.460(80)$ & $0.45(10)$ & $0.460(40)$ & $-0.36(14)$ & $-1.00(30)$ & $-0.14(11)$ & $-0.080(29)$ & $0.011(18)$ & $-0.048(20)$ & $-0.007(15)$ \\
\hline$\chi_{5,0,1}$ & $0.075(75)$ & $0.19(15)$ & $0.00(20)$ & $0.20(15)$ & $-0.15(43)$ & $0.00(50)$ & $-0.06(46)$ & $-0.32(11)^{\prime}$ & $0.090(99)$ & $0.047(85)$ & $0.076(64)$ \\
\hline$\chi_{5,1,0}$ & $0.140(20)$ & $0.260(50)$ & $0.250(70)$ & $0.320(90)$ & $0.18(33)$ & $-0.80(35)$ & $-0.27(27)$ & $-0.077(66)$ & $0.067(42)$ & $0.002(41)$ & $-0.078(34)$ \\
\hline$\chi_{6,0,0}$ & $4.0(5)$ & $6(1)$ & $4.67(43)$ & $3.7(2.0)$ & $-1.8(1.5)$ & $-4.1(1.5)$ & $-2.3(1.4)$ & $-0.94(42)$ & $0.14(28)$ & $0.96(25)$ & $0.42(21)$ \\
\hline
\end{tabular}

TABLE VII. Same as in Table IV for the eighth-order susceptibilities.

\begin{tabular}{lcc}
\hline \hline$T[\mathrm{MeV}]$ & 135 & 143 \\
\hline$\chi_{0,0,8}$ & $3.0(1.5)$ & $2.5(1.5)$ \\
$\chi_{1,0,7}$ & $0.50(50)$ & $0.90(35)$ \\
$\chi_{1,1,6}$ & $-0.15(15)$ & $0.00(20)$ \\
$\chi_{2,0,6}$ & $-0.05(15)$ & $0.25(13)$ \\
$\chi_{2,1,5}$ & $0.030(30)$ & $0.00(10)$ \\
$\chi_{2,2,4}$ & $0.000(40)$ & $0.031(21)$ \\
$\chi_{3,0,5}$ & $0.000(20)$ & $-0.07(11)$ \\
$\chi_{3,1,4}$ & $0.000(20)$ & $0.002(15)$ \\
$\chi_{3,2,3}$ & $-0.025(35)$ & $0.025(17)$ \\
$\chi_{3,3,2}$ & $0.000(25)$ & $-0.007(21)$ \\
$\chi_{4,0,4}$ & $0.10(10)$ & $0.100(70)$ \\
$\chi_{4,1,3}$ & $0.050(50)$ \\
$\chi_{4,2,2}$ & $0.000(50)$ & $0.070(45)$ \\
$\chi_{4,3,1}$ & $-0.0050(50)$ & $-0.020(40)$ \\
$\chi_{4,4,0}$ & $0.00(5)$ & $0.60(15)$ \\
$\chi_{5,0,3}$ & $0.15(20)$ \\
$\chi_{5,1,2}$ & $0.35(10)$ & $-0.050(50)$ \\
$\chi_{5,2,1}$ & $0.00(10)$ & $0.050(60)$ \\
$\chi_{5,3,0}$ & $0.025(75)$ & $0.26(14)$ \\
$\chi_{6,0,2}$ & $-0.020(40)$ & $0.35(10)$ \\
$\chi_{6,1,1}$ & $0.150(60)$ & $0.00(10)$ \\
$\chi_{6,2,0}$ & $0.30(12)$ & $0.95(30)$ \\
$\chi_{7,0,1}$ & $0.00(30)$ & $0.70(26)$ \\
$\chi_{7,1,0}$ & $0.70(20)$ & $35(10)$ \\
$\chi_{8,0,0}$ & $0.75(75)$ & \\
\hline \hline & $0.35(25)$ & $20(4)$ \\
\hline
\end{tabular}

(statistical + systematic) is taken into account, so that the choice of equally distributed points still seems a reasonable one.

The complete list of susceptibilities determined on the $32^{3} \times 8$ lattice are reported in Tables IV-VII, while in Figs. 10,12-15 some of those susceptibilities are shown, as a function of $T$, and compared with results obtained by other groups using the direct computation approach. We note that conserved charge susceptibilities are linked to the

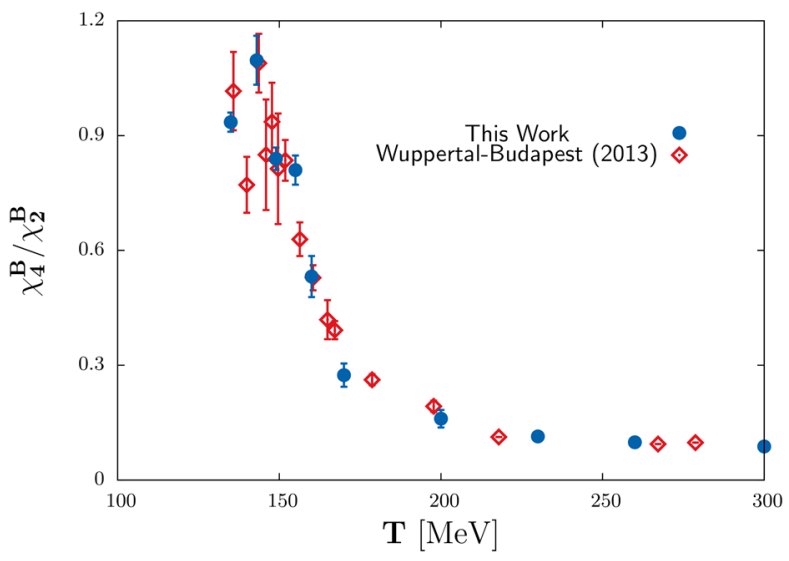

FIG. 10. Temperature dependence of the ratio $\chi_{4}^{B} / \chi_{2}^{B}$ of baryonic cumulants. Blue points correspond to our determinations while red points, corresponding to data obtained on a $N_{t}=8$ lattice using our own discretization, are taken from [7]. 


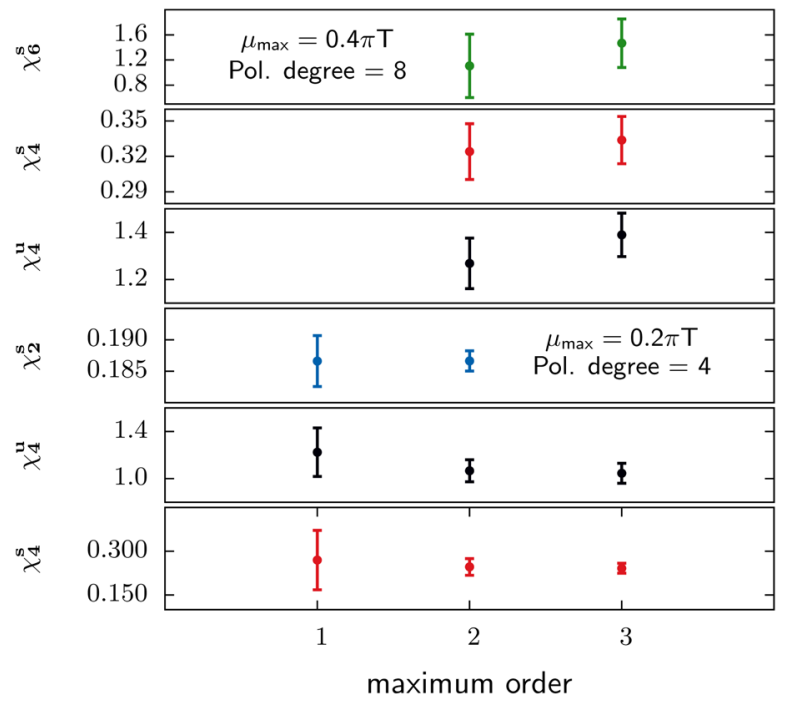

FIG. 11. We show how the precision attained for some susceptibilities changes when adding more and more cumulants to the global fit. The first three graphs correspond to a global fit performed using $\mu_{\max } / T=0.4 \pi$ and a polynomial of degree 8 , whereas for the last three $\mu_{\max } / T=0.2 \pi$ and a polynomial of degree 4 has been used. Data refer to simulations at $T=143 \mathrm{MeV}$.

quark number ones via linear relations [see Eq. (7)]. Correlations among quark number susceptibilities, as determined from the global fit procedure, turned out to be, in most cases, smaller than $10-15 \%$. Therefore the errorbars shown in Figs. 10,12-14 were computed by using Gaussian error propagation formulae. A very good agreement is found for almost all quantities and a higher precision is reached in our case, at least in the confined phase $T<T_{c}$. Only a small discrepancy is observed for the $\chi_{2}^{u s}$ in the high temperature regime (see Fig. 15). The source of this mismatch can be attributed to the different aspect

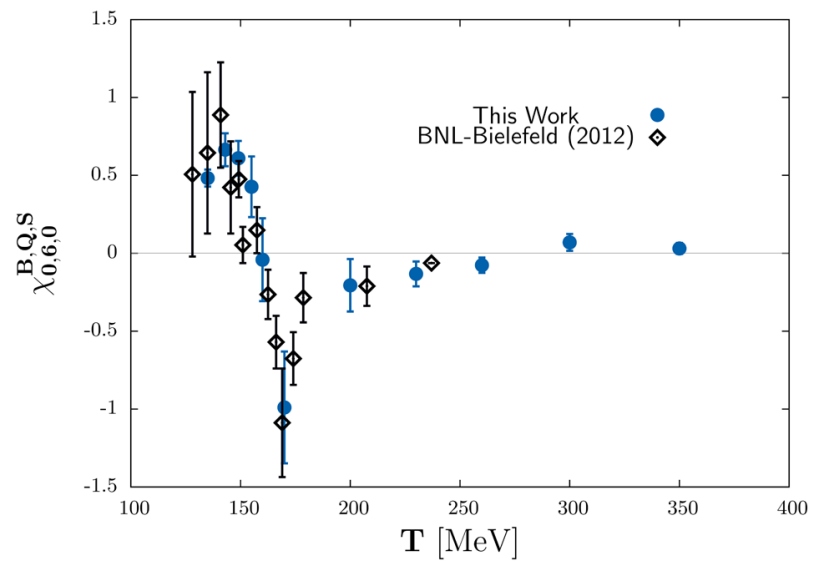

FIG. 12. Comparison of results on sixth-order cumulant of the electric charge fluctuations between this work and Ref. [46]. Diamonds refer to the determinations of Ref. [46] obtained on a $N_{t}=8$ lattice with the highly improved staggered quark (HISQ) action and almost physical quark masses.

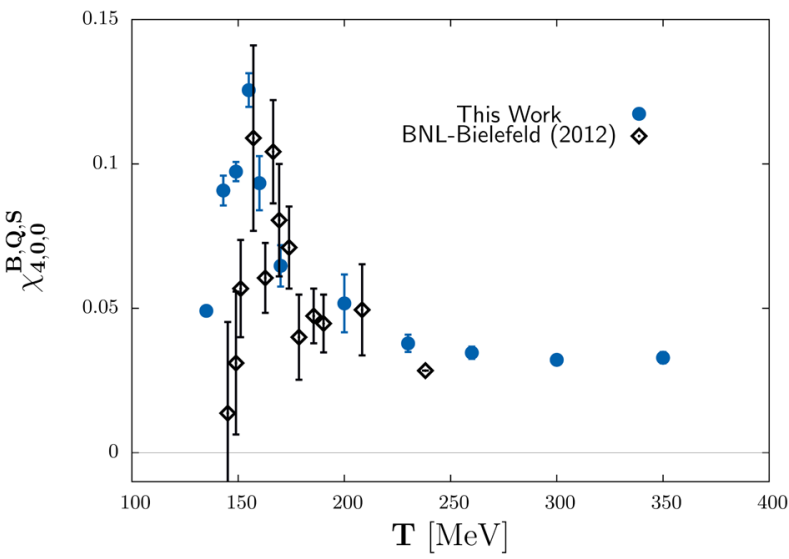

FIG. 13. Same as in Fig. 12 for the fourth-order cumulant of the net baryon number fluctuation.

ratios used in the two cases. Indeed, Ref. [45] adopted $N_{s} / N_{t}=3$, while in our case we have $N_{s} / N_{t}=4$; looking at Fig. 9 it is clear that finite volume effects are still nonnegligible for aspect ratio 3 and for this values of the temperature, and point exactly in the direction of the observed discrepancy.

\section{An application to the search for a critical end point}

The obtained susceptibilities could be used for several phenomenological analyses, like a determination of the freeze-out line [6-8]. However, since our results still lack of a reliable continuum extrapolation and have been obtained essentially for one single value of $N_{t}$, we prefer to postpone this to a future investigation.

There is, however, one kind of analysis which is worth doing even for a single value of $N_{t}$, and regards the possible emergence of a critical behavior for some value of the (real) baryon chemical potential, i.e. the existence and location of the critical end point. Indeed, high-order cumulants of the net baryon number distribution can be used to find signals of critical behavior in the $T-\mu_{B}$ plane, following the

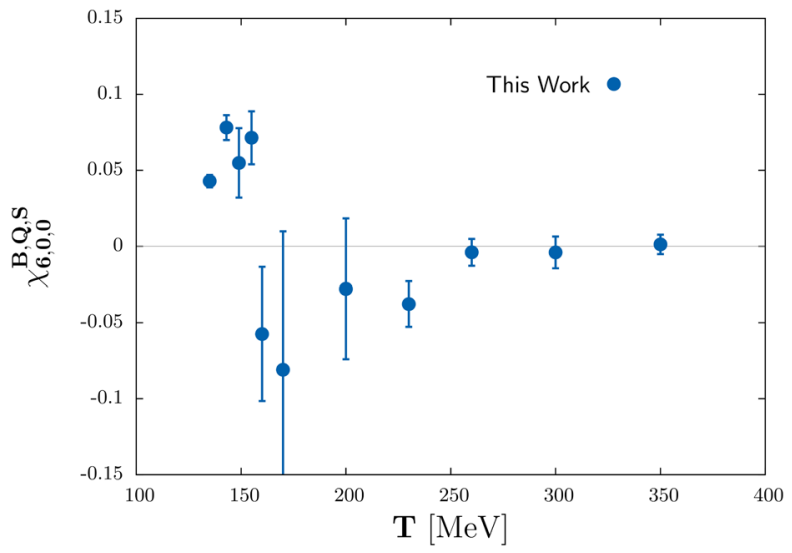

FIG. 14. Our determination of the sixth-order cumulant of the net baryon number fluctuation. 


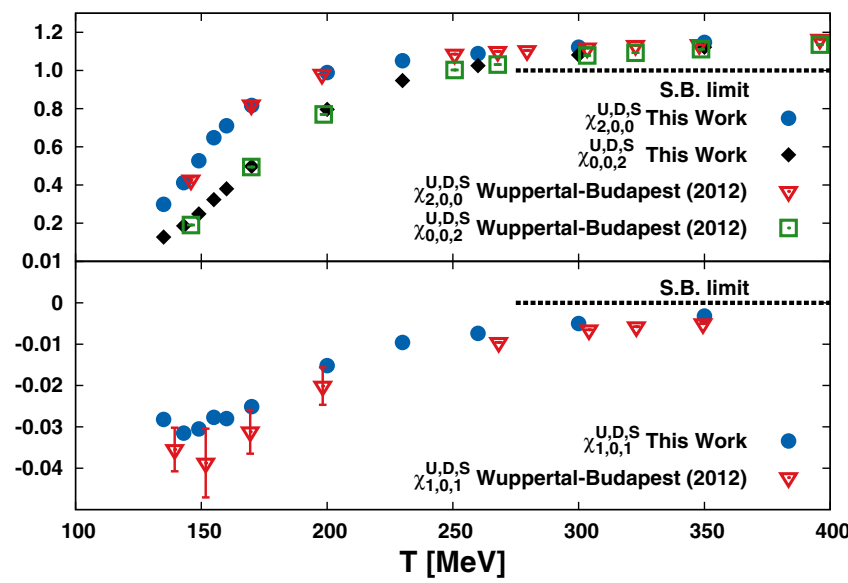

FIG. 15. Comparison between results on second-order susceptibilities obtained in this work and in [45] on the $N_{t}=8$ lattice and with our own discretization.

strategy of Refs. [12-14]. Setting $\mu_{u}=\mu_{d}=\mu_{s}=\mu_{B} / 3$ in the free energy expansion (1) we are left with a power series in the baryon chemical potential [see Eq. (7)]:

$$
\mathcal{F}\left(T, \mu_{B}\right)=\mathcal{F}(T, 0)+V T^{4} \sum_{n} \frac{\chi_{2 n}^{B}}{(2 n) !}\left(\mu_{B} / T\right)^{2 n}
$$

An example of the expansion is reported in Fig. 16, where we show, for a single value of the temperature, our lattice determination of the baryon number density (as a function of $\mu_{B}^{I}$ ), along with the various polynomial truncations of different orders coming from its Taylor expansion around $\mu_{B}^{I}=0$.

At a second-order $\mu_{B}$-driven phase transition, the free energy develops a nonanalyticity while the baryon number susceptibility $\chi_{2}^{B}$ shows a divergence. Therefore, signals of critical behavior can be inferred by looking for the radius of

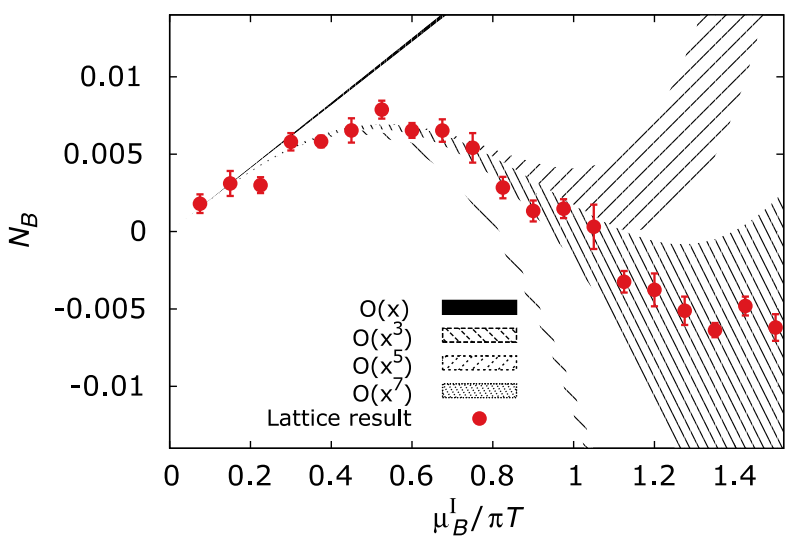

FIG. 16. Our lattice determination of the baryon number density as a function of the imaginary (baryon-)chemical potential is shown. Bands correspond to polynomial truncations at various orders of the series expansion around $\mu_{B}=0$. Data refer to $T=135 \mathrm{MeV}$. convergence of their Taylor series. To be physical, the singularity must be placed on the real $\mu_{B}$ axis, thus for the method to be effective the series must have only positive non-null terms. In this case, estimates for the radius of convergence of the free energy $\left(\rho^{f}\right)$ or of the baryon susceptibility $\left(\rho^{\chi}\right)$ are provided by:

$$
\rho_{n, m}^{f}=\left(\frac{\chi_{n}^{B} / n !}{\chi_{m}^{B} / m !}\right)^{\frac{1}{(m-n)}} \rho_{n, m}^{\chi}=\left(\frac{\chi_{n}^{B} /(n-2) !}{\chi_{m}^{B} /(m-2) !}\right)^{\frac{1}{(m-n)}}
$$

and they all coincide when the infinite $m$ and/or $n$ limit is taken. In our case, by using the few number of coefficients at our disposal a consistent determination of the critical end point requires that all the estimators in Eq. (16) agree with each other or at least show some signal of convergence. Of course, the number of terms needed to have such convergence is not known a priori and depends on the nature of the critical point, if it exists. However, we tested the possibility of finding the critical point using this method by bulding up a simple statistical toy model, the interested reader will find more details in the Appendix.

Since the pseudocritical line bends down for real baryon chemical potentials, the critical end point, if any, is expected for temperatures $T \leq T_{c} \sim 155 \mathrm{MeV}$. Hence, we evaluated the estimators in Eq. (16) using the susceptibilities up to $\chi_{8}^{B}$ for $T=135,143 \mathrm{MeV}$, and up to $\chi_{6}^{B}$ for $T=149,155 \mathrm{MeV}$. For this values of temperature all the determined $\chi_{2 n}^{B}$ appear to be greater than zero hence allowing for such a kind of analysis. The panels in Fig. 17 display our determinations, where they are also compared to the same quantities as extracted from a simple HRG model, where

$$
F\left(T, \mu_{B}\right)_{H R G}=A(T)+B(T) \cosh \left(\frac{\mu_{B}}{T}\right)
$$

and of course the asymptotic radius of convergence is infinite. As it can be noticed, the estimated radii do not

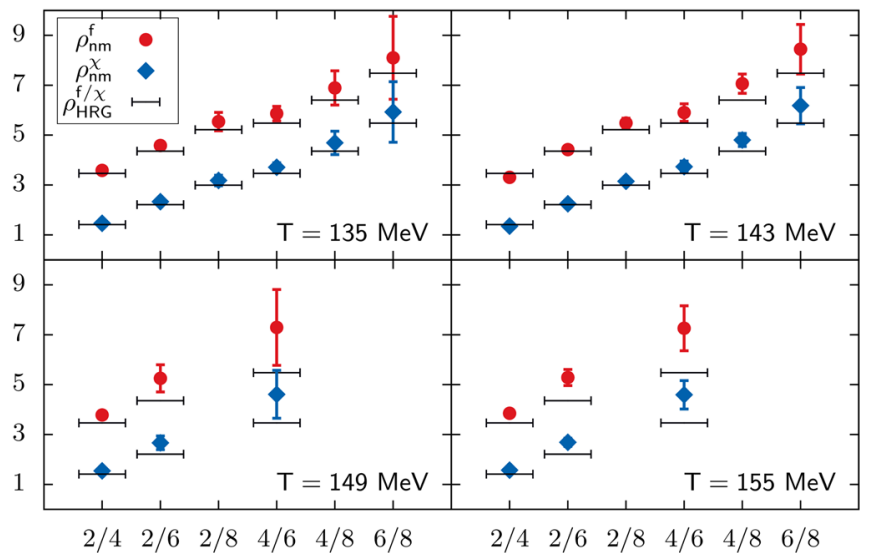

FIG. 17. Radius of convergence estimates for various temperatures below $T_{c}$. Circles/diamonds correspond to our estimate for $\rho_{n, m}^{f / \chi}$ while black lines are values predicted from the HRG model. 


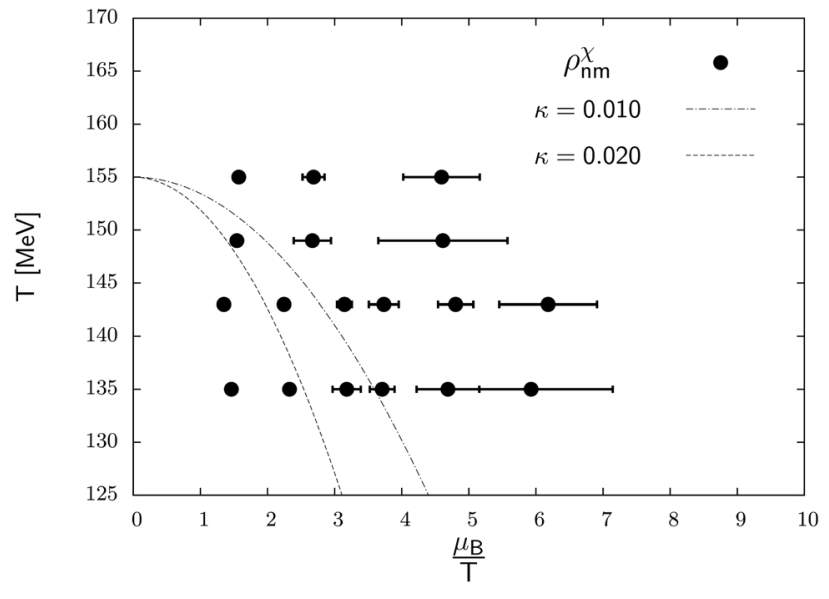

FIG. 18. The values of $\rho_{n, m}^{\chi}$ are shown along with the $\mathcal{O}\left(\mu_{B}^{2}\right)$ determination for the pseudocritical chiral line.

seem to converge to constant values as the order increases, but rather they are in good agreement with HRG estimates for $T<0.95 T_{c}$. For $T \gtrsim 0.95 T_{c}$, deviations are visible; however, they correspond to estimated radii which are larger than the HRG expectation.

The critical end point should be located somewhere along the pseudocritical starting from $\mu_{B}=0$. Therefore, it is interesting to report our estimated radii in the phase diagram together with the pseudocritical line as estimated from its curvature at $\mu_{B}=0$, i.e.

$$
\frac{T_{c}\left(\mu_{B}\right)}{T_{c}}=1-\kappa\left(\frac{\mu_{B}}{T_{c}}\right)^{2}+O\left(\mu_{B}^{4}\right) .
$$

This is shown in Fig. 18, where a range of values of $\kappa$ is reported, going from 0.010 to 0.020 , which roughly corresponds to the indications from most recent lattice determinations [44,47-51]. The estimated radii rapidly exceed, as the order in the expansion grows, the position of the estimated crossover line.

Therefore, we conclude that the present indication is that either no criticality appears at these temperatures, or that higher-order coefficients would be required in order to be sensible to the singular part of the free energy. We stress that to put this result on more solid grounds, either in favor or in disfavor of a CEP at these temperatures, several successive coefficients $\chi_{n}^{B}$ could be required. ${ }^{3}$ Moreover, the same analysis should be repeated for different values of $N_{t}$ to control UV cutoff effects.

\footnotetext{
${ }^{3}$ As example, in Ref. [52] the authors tried to determine the Critical Point of the 3D Ising Model $\left(T_{c}, H_{c}=0\right)$ putting an external magnetic field $H_{o}$ and then evaluating several cumulants of the free energy Taylor expansion in $\frac{\left(H-H_{o}\right)}{T}$ at fixed temperature. They found that an accurate determination of the Critical Point $\left(H_{c} \approx 0\right)$ by means of radius of convergence estimates, requires the evaluation of at least 8 coefficients in the cumulant expansion.
}

\section{DISCUSSION AND CONCLUSIONS}

In this work we studied $N_{f}=2+1$ QCD by means of analytic continuation from three different imaginary chemical potentials coupled to the up, down and strange quarks. We performed simulations for 11 values of the temperature, using a $32^{3} \times 8$ lattice with a stout staggered fermion discretization, the tree level Symanzik improvement for the pure gauge part and physical quark masses. First- and second-order free energy derivatives were measured as a function of the purely imaginary chemical potentials, and then interpolated by means of polynomial functions in order to reconstruct the Taylor expansion of the free energy around $\left\{\mu_{i}\right\}_{i=u, d, s}=0$. The chosen trajectories in the imaginary $\left\{\mu_{i}\right\}_{i=u, d, s}$ space [see Eq. (13)] ensure the possibility to estimate all kind of fluctuations and cross-correlations among conserved charges up to order eight. Different ranges of chemical potentials and different polynomials have been used, in order to monitor systematic effects related to analytic continuation. Different spatial sizes have been also investigated, both below and above $T_{c}$, obtaining as a result that finite size effects are well under control if an aspect ratio at least 4 is used. No systematic analysis has been performed regarding UV cutoff effects: our results are mostly limited to $N_{t}=8$ lattices and a continuum extrapolation is postponed to a future investigation.

One of the main purposes of this study was that of checking the efficiency of the method, as compared with the standard determination of nonlinear susceptibilities from simulations at zero chemical potentials, and give indications about the optimal strategy to be followed. We provided susceptibilities up to order six for nine values of temperature and up to order eight for $T=135,143 \mathrm{MeV}$, where the extended range of measurements at imaginary $\mu\left(\mu_{I, \max }=0.8 \pi / T\right)$ allowed us to fit polynomials up to order ten. Our results are in good agreement with previous standard determinations. Regarding efficiency, we obtained that analytic continuation can lead to a significant improvement below the pseudocritical temperature $T_{c}$ : in terms of computational cost, this improvement is of order ten for fourth-order diagonal light quark susceptibilities, and goes up to a factor 100 for nondiagonal ones; we could not make a direct test for higher-order susceptibilities, for which the improvement is expected to be even larger.

On the contrary, analytic continuation does not reveal to be a competitive strategy above $T_{c}$. One possible reason is related to the restoration of chiral symmetry, which causes a significant reduction in the statistical fluctuations present in the noisy estimators and in the numerical cost of matrix inversions: both these factors go in the direction of a strong improvement in the standard determination. Another possible reason is related to the reduced range of explorable imaginary chemical potentials, due to RW or RW-like transitions, which affects both the statistical accuracy of the global fit and the systematic uncertainty related to truncation effects. 
The precision reached below $T_{c}$ allowed us to perform an analysis regarding the possible location of the CEP. We evaluated cumulants of the net baryon number fluctuations for four values of $T \leq T_{c}$ : up to $\chi_{8}^{B}$ for $T=135,143 \mathrm{MeV}$ and up to $\chi_{6}^{B}$ for $T=149,155 \mathrm{MeV}$. Various estimators of the radius of convergence of the Taylor expansion, both for the free energy and for the baryon susceptibility, have been considered. We did not observe any signal of convergence of the estimated radii, and for $T \lesssim 0.95 T_{c}$ the estimates are consistent with a HRG-like behavior. Moreover, the estimated radii go well beyond the estimated location of the pseudocritical line as the order of the estimator increases. We retain that this result could be interpreted in two possible ways:

(i) No critical end point exists, at least for the discretization of QCD adopted in the present study, and within the explored range of temperatures;

(ii) sixth/eighth-order baryon number susceptibilities are still not sufficient to be sensitive to the singular part of the free energy; moreover, the critical end point could be located for large chemical potentials, for which present lattice methods, which work well for small values of $\mu_{B} / T$, are not well suited.

Finally, let us discuss how our results and the method could be improved in future studies, especially in view of an extension to finer lattices, in order to perform a continuum extrapolation. An outcome of our investigation is that the information on quadratic susceptibilities allows to achieve a much better overall accuracy on the global fit, and a significant improvement with respect to the measurement of quark number densities only, whereas the inclusion of third-order cumulants does not have a significant impact. This is important in order to define a strategy aimed at computing higher order susceptibilities. Indeed, looking at Figs. 1-4, we see that the number and the order of nonlinear susceptibilities which one is able to determine can be increased by either increasing the number of measured susceptibilities, or by increasing the number of trajectories in the chemical potentials space along which the simulation points are taken. However, in view of the difficulty in adding statistically significant information by measuring third-order susceptibilities, the suggested strategy for the future is to measure directly free energy derivatives up to order two and to add more trajectories of simulated points. For instance, adding two more lines to Eq. (13) (see Fig. 4), corresponding to an increase in computational effort of about $1 / 3$, would allow to completely determine susceptibilities up to order 12 .

\section{ACKNOWLEDGMENTS}

We thank Claudio Bonati, Philippe de Forcrand and Ettore Vicari for useful discussions. F. S. received funding from the European Research Council under the European Community Seventh Framework Programme (FP7/20072013) ERC Grant Agreement No. 279757. Simulations have been performed on the BlueGene/Q Fermi at CINECA (Projects Iscra-B/RENQCD and INF16_npqcd).

\section{APPENDIX: A SIMPLE STATISTICAL TOY MODEL}

We tested the possibility of finding, by the radius of convergence estimate method, the location of a critical point, by using a simple test function (which plays the role of the baryon number density) with a nonanalyticity located at real chemical potential. We sampled this function and its first derivative on the imaginary $\mu$ axis, by adding a statistical Gaussian noise to the function values, in order to obtain data points with statistical errors, then trying to reconstruct the Taylor expansion around $\mu=0$ by means of a polynomial interpolation to the sampled data, adopting the same procedure for the estimate of statistical and systematic uncertainties adopted for the real QCD data.

We used as a test function

$$
n(\mu)=\frac{\mu}{\mu_{c}^{2}-\mu^{2}}
$$

with $\mu_{c}=2.5$. This function and its first derivative, which plays the role of the second-order baryon susceptibility, were sampled in the range $0 \leq \mu_{I}=\operatorname{Im}(\mu) \leq 1.5$. To determine systematic errors we exactly followed the guidelines used for quark number susceptibilities and fitted the sampled data with polynomials up to order 12. As for the quark number susceptibilities the very last term of the highest-order polynomial we used in the best fit procedure was not considered, because it might have a large uncontrolled bias due to truncation effects. Susceptibilities up to order ten were then used to compute the estimators in Eq. (16). Results are shown in Fig. 19.

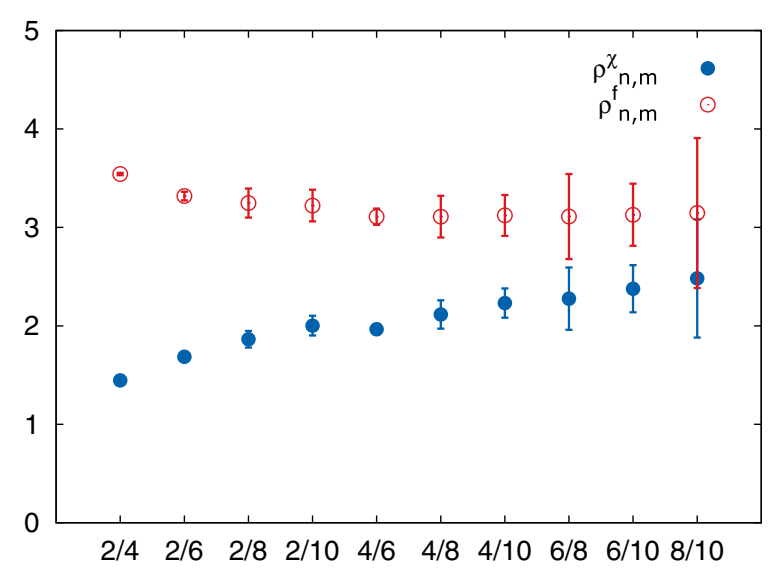

FIG. 19. Radius of convergence estimates for our statistical toy model. Filled points represent the radius of convergence estimates for the Taylor expansion of the susceptibility while the unfilled ones the estimates for the Taylor expansion of the free energy [see Eq. (16)]. 
As it is clear from the figure, the estimators seem to convergence to the correct value of $\mu_{c}=2.5$ with the estimators $\rho_{n, m}^{\chi}$ showing a faster convergence with respect to the $\rho_{n, m}^{f}$ 's. In spite of the simplicity of the statistical model, the important outcome is that it seems at least reasonable to perform, in the case of $\mathrm{QCD}$, such a kind of analysis, even though just a few number of susceptibilities are known. Of course, as we have already emphasized, the actual number of needed terms will depend on the particular critical behavior.
[1] Y. Aoki, G. Endrodi, Z. Fodor, S. D. Katz, and K. K. Szabo, Nature (London) 443, 675 (2006).

[2] M. M. Aggarwal et al. (STAR Collaboration), Phys. Rev. Lett. 105, 022302 (2010).

[3] X. Luo (STAR Collaboration), Proc. Sci., CPOD2014 (2015) 019 [arXiv:1503.02558].

[4] L. Adamczyk et al. (STAR Collaboration), Phys. Rev. Lett. 113, 092301 (2014).

[5] A. Adare et al. (PHENIX Collaboration), Phys. Rev. C 93, 011901 (2016)

[6] A. Bazavov et al., Phys. Rev. Lett. 109, 192302 (2012).

[7] S. Borsanyi, Z. Fodor, S. D. Katz, S. Krieg, C. Ratti, and K. K. Szabo, Phys. Rev. Lett. 111, 062005 (2013).

[8] S. Borsanyi, Z. Fodor, S. D. Katz, S. Krieg, C. Ratti, and K. K. Szabo, Phys. Rev. Lett. 113, 052301 (2014).

[9] C. R. Allton, S. Ejiri, S. J. Hands, O. Kaczmarek, F. Karsch, E. Laermann, C. Schmidt, and L. Scorzato, Phys. Rev. D 66, 074507 (2002).

[10] C. R. Allton, S. Ejiri, S. J. Hands, O. Kaczmarek, F. Karsch, E. Laermann, and C. Schmidt, Phys. Rev. D 68, 014507 (2003).

[11] R. V. Gavai and S. Gupta, Phys. Rev. D 68, 034506 (2003).

[12] R. V. Gavai and S. Gupta, Phys. Rev. D 71, 114014 (2005).

[13] R. V. Gavai and S. Gupta, Phys. Rev. D 78, 114503 (2008).

[14] S. Datta, R. V. Gavai, and S. Gupta, Nucl. Phys. A904-905, 883c (2013).

[15] A. Milchev, K. Binder, and D. W. Heermann, Zeitschrift für Physik B Condensed Matter 63, 521 (1986).

[16] P. de Forcrand and O. Philipsen, Nucl. Phys. B642, 290 (2002).

[17] P. de Forcrand and O. Philipsen, Nucl. Phys. B673, 170 (2003).

[18] M. D'Elia and M.-P. Lombardo, Phys. Rev. D 67, 014505 (2003).

[19] M. D'Elia and M. P. Lombardo, Phys. Rev. D 70, 074509 (2004).

[20] P. Cea, L. Cosmai, M. D’Elia, and A. Papa, J. High Energy Phys. 02 (2007) 066.

[21] M. D’Elia, F. Di Renzo, and M. P. Lombardo, Phys. Rev. D 76, 114509 (2007).

[22] M. D'Elia and F. Sanfilippo, Phys. Rev. D 80, 014502 (2009).

[23] T. Takaishi, P. de Forcrand, and A. Nakamura, Proc. Sci., LAT2009 (2009) 198 [arXiv:1002.0890].

[24] J. Gunther, R. Bellwied, S. Borsanyi, Z. Fodor, S. D. Katz, A. Pasztor, and C. Ratti, EPJ Web Conf. 137, 07008 (2017).

[25] H. Panagopoulos and E. Vicari, J. High Energy Phys. 11 (2011) 119.
[26] C. Bonati, M. D’Elia, and A. Scapellato, Phys. Rev. D 93, 025028 (2016).

[27] C. Bonati, M. D’Elia, P. Rossi, and E. Vicari, Phys. Rev. D 94, 085017 (2016).

[28] P. Weisz, Nucl. Phys. B212, 1 (1983).

[29] G. Curci, P. Menotti, and G. Paffuti, Phys. Lett. B 130, 205 (1983); 135, 515 (1984).

[30] C. Morningstar and M. J. Peardon, Phys. Rev. D 69, 054501 (2004).

[31] P. Hasenfratz and F. Karsch, Phys. Lett. B 125, 308 (1983).

[32] R. V. Gavai, Phys. Rev. D 32, 519 (1985).

[33] R. V. Gavai and S. Sharma, Phys. Lett. B 749, 8 (2015).

[34] M. A. Clark, A. D. Kennedy, and Z. Sroczynski, Nucl. Phys. B, Proc. Suppl. 140, 835 (2005).

[35] M. A. Clark and A. D. Kennedy, Phys. Rev. Lett. 98, 051601 (2007).

[36] M. A. Clark and A. D. Kennedy, Phys. Rev. D 75, 011502 (2007).

[37] B. Efron, Ann. Stat. 7, 1 (1979).

[38] Y. Aoki, S. Borsanyi, S. Durr, Z. Fodor, S. D. Katz, S. Krieg, and K. K. Szabo, J. High Energy Phys. 06 (2009) 088.

[39] S. Borsanyi, G. Endrodi, Z. Fodor, A. Jakovac, S. D. Katz, S. Krieg, C. Ratti, and K. K. Szabo, J. High Energy Phys. 11 (2010) 077.

[40] S. Borsanyi, Z. Fodor, C. Hoelbling, S. D. Katz, S. Krieg, and K. K. Szabo, Phys. Lett. B 730, 99 (2014).

[41] A. Roberge and N. Weiss, Nucl. Phys. B275, 734 (1986).

[42] C. Bonati, M. D'Elia, M. Mariti, M. Mesiti, F. Negro, and F. Sanfilippo, Phys. Rev. D 93, 074504 (2016).

[43] P. Cea, L. Cosmai, M. D'Elia, C. Manneschi, and A. Papa, Phys. Rev. D 80, 034501 (2009).

[44] C. Bonati, M. D'Elia, M. Mariti, M. Mesiti, F. Negro, and F. Sanfilippo, Phys. Rev. D 90, 114025 (2014).

[45] S. Borsanyi, Z. Fodor, S. D. Katz, S. Krieg, C. Ratti, and K. Szabo, J. High Energy Phys. 01 (2012) 138.

[46] C. Schmidt (BNL-Bielefeld Collaboration), Nucl. Phys. A904-905, 865c (2013).

[47] C. Bonati, M. D'Elia, M. Mariti, M. Mesiti, F. Negro, and F. Sanfilippo, Phys. Rev. D 92, 054503 (2015).

[48] P. Cea, L. Cosmai, and A. Papa, Phys. Rev. D 89, 074512 (2014).

[49] P. Cea, L. Cosmai, and A. Papa, Phys. Rev. D 93, 014507 (2016).

[50] P. Hegde and H.-T. Ding (Bielefeld-BNL-CCNU Collaboration), Proc. Sci., LATTICE2015 (2016) 141.

[51] G. Endrodi, Z. Fodor, S. D. Katz, and K. K. Szabo, J. High Energy Phys. 04 (2011) 001.

[52] M. A. York and G. D. Moore, arXiv:1106.2535. 